\title{
Stochastic Poisson Game for an Online Decentralized and Coordinated Parking Mechanism
}

\author{
Lili Du ${ }^{1}$, Siyuan Gong ${ }^{2}$
}

\begin{abstract}
:
This paper proposes a decentralized and coordinated online parking mechanism (DCPM), which seeks to reduce parking congestion at multiple parking facilities in a central business district (CBD) through guiding the parking decisions of a parking coordination group. To establish this DCPM, this study develops a stochastic Poisson game to model the competitions among parking vehicles en route at multiple parking facilities. The equilibrium condition for the proposed stochastic Poisson game is formulated through involving travelers' parking choice behavior described by multinomial logit model. Furthermore, we prove that the stochastic Poisson game is a potential game with a unique equilibrium. A simultaneously updating distributed algorithm is developed to search the equilibrium solution of the DCPM. Its convergence is proved by both mathematical analysis and numerical experiments. The numerical experiments are conducted to test the efficiency of the DCPM, based on a real-world CBD covering Guicheng Community, Nanhai District at Foshan in China. The performance of the DCPM is compared to three greedy strategies following the nearest first, cheapest first, and least cruise first policies, respectively. The experimental results demonstrate that the DCPM significantly reduces cruise vehicles and average cruise distance per vehicle from all other three greedy strategies; the least cruise first strategy, which takes advantage of the real-time open spots information at parking facilities, performs better than the nearest first and the cheapest first strategies without the access to real-time information. The DCPM can further improve the benefit of the real-time information. Additionally, in terms of walking distance and parking cost, the DCPM provide a trade-off solution between the nearest first and the cheapest first strategies.
\end{abstract}

Keywords: parking, stochastic Poisson game, equilibrium, distributed algorithm

\footnotetext{
${ }^{1}$ Corresponding author, Department of Civil, Architectural and Environmental Engineering, Illinois Institute of Technology, 3201 South Dearborn Street, Chicago, Illinois 60616 U.S.A., Email: ldu3@iit.edu

${ }^{2}$ Department of Civil, Architectural and Environmental Engineering, Illinois Institute of Technology, 3201 South Dearborn Street, Chicago, Illinois 60616 U.S.A., Email: sgong1@ @awk.iit.edu
} 


\section{Introduction}

Existing studies indicate that around $30 \%$ of the traffic in dense populated downtown is cruising for parking, and a significant part of the trips is searching for a parking space, which causes extra travel time, consumes road capacity, burns extra fuel, and produces toxic emissions (e.g. cars cruising for parking burned 47,000 gallons of gasoline and produced 730 tons of carbon dioxide over one year in a small Los Angeles business district (Shoup, 2006)). Thus, developing efficient parking mechanisms is imperative. Recently, advances in wireless communication and sensing technologies have enabled efficient parking space monitoring and parking information provision systems. This technology advances paved the way toward smart parking assistance systems, which provide parking guidance with the aim to reduce cruise and related traffic congestion.

The proposed research shares the same interests with some existing research but notices that existing independent parking decisions (IP) (Thompson and Richardson, 1998; Lam et al., 2006; Li et al., 2007; Liu et al., 2012; Kokolaki et al., 2014; Schlote et al, 2014; Mackowski, et al., 2015), or centralized reservation strategies (CP) (Caliskan et al., 2006; Teodorović and Lučić, 2006; Delot et al., 2009 and 2013; Geng and Cassandras, 2013; Zou et al., 2015; Chen et al., 2015) in the literature will meet obstacles to function efficiently. More exactly, as most of the travelers in the traffic use the same parking assistant system, IP strategy will suffer from the conflict between the system performance and individual parking interests (Kokolaki et al. 2014). Namely, when most of parking travelers obtain and respond to the same real-time parking information independently, it is very likely that these travelers may select the same parking facilities (such as the cheapest one, the closest one, or the one with more open spots) in a short time period. Then, the collective effect of these common actions will cause parking congestion at the selected parking facility and lead to cruising for those vehicles which do not find an open spot. This type of congestions may oscillate among candidate parking facilities along with the information update over time. On the other hand, centralized parking reservation strategy (such as Chen et al., 2015) may address this conflict, but they have the obstacles to work efficiently for a large scale of parking demands in a central business district of a metropolitan, which usually involves multiple parking facilities belongs to different private agents and a large volume of users which comes randomly. In addition, parking reservation may limit the flexibility of trips or cause the waste of some parking spots to some extent. The state-of-the-art shows that it is still an open problem about how to manage a large scale of online parking demands. The proposed research is motivated by and seeks to address this problem from a different angle.

This study works on the parking decision process of a group of travelers in a central business district (CBD) including multiple parking facilities. We consider that establishing coordination rather than allowing pure competition among parking customers represents a potentially efficient management scheme. Along with this idea, a decentralized and coordinated online parking mechanism (DCPM) is developed for a group of parking travelers which compete in multiple parking facilities in a CBD in a time interval. Specifically, we view travelers as rational selfish agents, which select parking facility aiming to maximum its utility; moreover, they are willing to share tentative parking preference under privacy protection schemes (Jung et al., 2013). Intelligent parking facilities are well monitored and are able to disseminate real-time parking service related information such as the number of open spots to travelers, who are searching for parking space nearby. Under this mechanism, a number of smart vehicles/travelers make parking decisions as they are close to their respective destinations. Each of them joins an online parking coordination group (PCG), in which each smart traveler decides its parking priority among the candidate parking facilities by repeatedly proposing its tentative parking priority to all other travelers, and then updating it according to other travelers' parking choice decisions, parking facilities' occupancy, walking time, and pricing information until a resolution (i.e. an equilibrium parking decision (EPD)) among all travelers is reached. Parking vehicles will choose a parking lot according to the parking priority of an EPD. Parking vehicles unallocated in last time interval may choose to join a PCG in next time interval or change their trip plan without parking needs in this CBD area. Thus, the number of travelers (i.e. smart vehicles) in each PCG is assumed to be uncertain. Note that smart vehicles and travelers are equivalent in this paper. We use them interchangeably to fit the context hereafter. 
This DCPM aims to reduce the competition among vehicles searching for parking spaces, so that we can mitigate the congestion at the parking facilities while sustain individual travelers' parking interests. At the same time, it does not cause extra charges to the drivers. The proposed mechanism can be implemented on autonomous vehicles or smartphones as a software agent to assist parking space search. Namely, the parking decision is made automatically by the software rather than by humans themselves.

Mathematically, this study models the proposed DCPM as a Poisson game integrating stochastic user behavior (shortened as stochastic Poisson game hereafter) based upon the Poisson Game proposed in (Myerson, 1998). More exactly, this study considers that the vehicles searching for parking spaces in a CBD during a time interval form one PCG. The vehicles in this PCG are grouped into different types in the game according to their destinations; all the vehicles in each type have the same parking priority to all the candidate parking facilities since their destinations are close. The number of vehicles joining each type (heading to a set of close destinations) in a time interval follows a Poisson distribution. The expected utility function, mainly factoring the probability that an arriving traveler has to perform cruise, is formulated using queueing theory, given the parking time obeys exponential distribution and the number of travelers arriving to a parking facility follows Poisson distribution too. Given that travelers' parking choice behavior is modeled by Multinomial Logit discrete choice model (Sheffi, 1985), this study formulates the equilibrium condition for the proposed game. Rigorous mathematical analysis proves the following critical characteristics of the stochastic Poisson game. First, the proposed stochastic model is a potential game, i.e., the equilibrium of the game is equivalent to the optimal solution of an optimization problem ${ }^{3}$ (Monderer and Shapley, 1996.). Second, the proposed stochastic model has a unique equilibrium parking decisions since the potential optimization has a unique optimization solution. Last, this study develops a simultaneously update distributed algorithms (SDA) to search the equilibrium parking decisions of the DCPM. We provide mathematical analysis to prove the convergence of the SDA to the equilibrium parking decision.

Numerical experiments are conducted based on a real-world CBD and its operational data. The DCPM strategy is compared to three greedy parking strategies, which guide the travelers following the nearest first, the cheapest first or the least cruise strategy, by which a traveler checks the availability of the parking facilities focusing on minimizing the walking distance, parking price, or cruise opportunity respectively. The performance of these strategies is compared from four perspectives. Regarding to the number of cruise vehicles and the average cruise distance per vehicle, the results demonstrate that the least cruise strategy with real-time open spots information at parking facilities performs better than the nearest first and the cheapest first strategies without real-time information provision. More importantly, the DCPM outperforms all three greedy strategies significantly, thus the DCPM will further improve the benefit of the real-time information. Additionally, regarding to the walking distance and parking cost per vehicle, the DCPM produces a trade-off solution as compared to the nearest first and the cheapest first strategies. Overall, the proposed DCPM can reduce the competition among vehicles on the parking resources and efficiently guide parking demands.

The rest of the paper is structured as follows. Section 2 reviews the relevant research in literature. Section 3 formulates the parking problem of interests. Section 4 first introduces the fundamental knowledge of the Poisson game. Based on that, this study proposes the stochastic Poisson game to formulate the DCPM, and develops its equilibrium formulation. Furthermore, we prove that the proposed stochastic Poisson game is a potential game. Section 5 designs a simultaneously updated distributed algorithm (SDA) to search for the equilibrium solution for the DCPM. The uniqueness of the equilibrium for the DCPM and the convergences of the SDA algorithm are proved by the mathematical deduction. Section 6 presents the numerical experiment and the obtained key results and insights. The summary of the research is given in Section 7.

${ }^{3}$ A game is said to be a potential game if the incentive of all players to change their strategy can be expressed using a single global function called the potential function. The objective function of the equivalent optimization model to the Stochastic Poisson game is just the global function to represent the incentive of all players for this study. 


\section{Literature Review}

Recently, the technology advances on wireless sensor, communication and connected vehicle systems have spurred extensive research interests on information-assisted parking search systems (see the review of Idris et al., (2009)). Quite a few researchers have noticed the effect of the competition among vehicles on parking resources, and proposed game theoretic approaches to address congestion issues at parking facilities (Ayala et al., 2011,2012a and 2012b; Gao et al., 2013; Kokolaki et al. 2014; Mejri et al., 2014; He et al., 2015; Mackowski, et al 2015). This study shares the same interests but proposes a different methodology. Below briefly reviews previous efforts and then presents the distinguishing features of this study.

There are a few efforts in the literature, which study the parking demand assignment under the competition situation without involving pricing strategies. For example, Ayala et al. first introduced game models into parking congestion issues by their seminal work in (Ayala et al., 2011 and 2012a). They proposed pure strategy congestion game models to analyze parking demand assignment in competitive settings. Both a centralized scheme and a distributed solution method are developed for this parking game, considering both complete and incomplete information scenarios. Mejri et al. (2014) proposed a full cooperation method (optimization approach) between parking coordinators which optimize the distribution of the parking slots, and a competition (game theoretic approach) method among vehicles searching for parking spaces. Their simulations indicate that the proposed framework outperforms the centralized model as well as greedy approaches. On the other hand, quite a few studies developed game theoretic models to address parking congestion issues involve pricing strategies. For example, Ayala et al. (2012b) developed a pure strategy Nash game model to address parking related traffic congestion issue, in which pricing strategy is set by using the parking availability information. Guo et al (2013) proposed two types of parking choice models, a static game theoretic model and a dynamic neo-additive capacity model, to capture the competition among drivers for limited desirable parking spaces. Their experiment results indicated that drivers' psychological characteristics towards uncertainty are significant factors in the parking choice decision-making process. Kokolaki et al. (2014) formulated the uncoordinated information-assisted parking search process as an instance of resource selection games, and systematically explored the efficiency of parking search in urban environments with two types of parking resource facilities: inexpensive but limited facilities (public) and expensive yet unlimited ones (private), assuming perfect knowledge of prices and costs, total parking capacities and demand. The authors also pointed out that a system optimal parking demand assignment may be approached by manipulating the price of the parking facilities. He et al. (2015) provided an uncooperative static atomic parking game with complete information to address parking competition issues. They discuss optimal pricing schemes that steer such parking competition to a system optimum assignment of parking spaces. Mackowski, et al (2015) developed a dynamic non-cooperative Stackelberg leader-follower game to set parking prices in real-time for effective parking access and space utilization.

Compared to all aforementioned efforts, this study is distinguished by the following aspects. First of all, the specifications of the game model in this research are very different. This study considers an uncertain number of vehicles competing on the parking facilities in a time interval, which may reflects the reality better since vehicles might change their trip plans in real-time. Accordingly, a Poisson game based game model with an uncertain number of players is developed in this study, rather than Nash game or congestion game considering a deterministic number of vehicles is adopted in most of previous studies. This difference leads to very different analyses. Second, this study integrates the choice model into Poisson game, proposes the stochastic Poisson game, and further formulates the equilibrium condition. Last, a distributed algorithm is developed to search for the equilibrium solution of the stochastic Poisson game and provides rigorous analysis on the convergence of the algorithm. To the best of our knowledge, all these research efforts are innovative to previous research. The proposed research will greatly contribute to the literature in this research area. 
3. Parking problem description

This research studies the parking decision process of a group of vehicles among multiple parking facilities in a central business district (CBD). The time period of interests is discretized into intervals of the same length $\Delta \tau$, say 10 minutes, indexed by $\tau=1, \ldots, \Xi$. There are $M$ parking facilities located at different locations, indexed by $m$, in the CBD. Each parking lot is able to detect the number of the open parking spots $n_{m}^{\tau}, m=1, \ldots, M$, and shares the occupancy information with vehicles en-route at the beginning of each time interval $\tau$. Clearly, $0 \leq n_{m}^{\tau} \leq N_{m}$, where $N_{m}$ is the capacity of the parking facility $m$. Suppose there are many points of interest (such as the final destinations of the drivers) within the CBD, we separate the vehicles/travelers into $V$ types indexed by $v$, according to their own destinations. Vehicles with close destinations will most likely be assigned into the same type and they will share the same preference in choosing parking facilities.

All the discussion below focuses on one time interval, thus the index of time interval $\tau$ is omitted. In a given time interval, the number of travelers in each type $v$ follows a Poisson distribution with the mean equal to $\bar{n}_{v}$. Let the probability that a traveler in type $v$ chooses parking facility $m$ be $p_{m}^{v}, \sum_{m=1}^{M} p_{m}^{v}=1$. Then, the number of travelers in type $v$ choosing a parking facility $m$ also follows a Poisson distribution with the mean equal to $\bar{n}_{v} p_{m}^{v}$. In turn, the total number of the travelers choosing parking facility $m$ also obeys a Poisson distribution with the mean equal to $\sum_{v=1}^{V} \bar{n}_{v} p_{m}^{v}$.

We also assume the time period that a vehicle stays in parking facility $m$ obeys an exponential distribution with the mean equal to $1 / \theta_{m}$. Hence, this study models the service of parking facility $m$ as an $M / M / c_{m}$ queue model (single queue, $c_{m}$ servers, with Poisson arrival, and exponential service time) with the arrival rate being:

$$
\lambda_{m}=\frac{1}{\Delta \tau} \sum_{v=1}^{V} \bar{n}_{v} p_{m}^{v}
$$

the service rate per spot at the facility $m$ being $\theta_{m}$, and the number of servers $c_{m}$ being the expected number of open spots. More exactly, at the beginning of time interval $\tau$, there are $n_{m}$ parking spots available and $o_{m}$ spots occupied at the parking facility $m$, and then on average there are $\Delta \tau o_{m} \theta_{m}$ parking spots released due to the departures during the time interval $\Delta \tau$, and the excepted number of open spots in facility $m$ is $c_{m}=n_{m}+\Delta \tau o_{m} \theta_{m}$ during a time interval $\Delta \tau$. Let $\mu_{m}=c_{m} \theta_{m}$. Accordingly, the probability that an arriving traveler has to join the queue (all parking spots are occupied and this vehicle need cruise) at a parking facility $m$ is given by Equation (2) (Gross et al., 2008).

$$
q_{m}= \begin{cases}\frac{\left(\frac{\left(c_{m} \rho_{m}\right)^{c_{m}}}{c_{m} !}\right)\left(\frac{1}{1-\rho_{m}}\right)}{\sum_{k=0}^{c_{m}-1} \frac{\left(c_{m} \rho_{m}\right)^{k}}{k !}+\left(\frac{\left(c_{m} \rho_{m}\right)^{c_{m}}}{c_{m} !}\right)\left(\frac{1}{1-\rho_{m}}\right)}, & \text { if } \rho_{m}<1 \\ 1, & \text { if } \rho_{m} \geq 1\end{cases}
$$

where $\rho_{m}=\frac{\lambda_{m}}{\mu_{m}}$. Note that $q_{m}$ is a function of $\lambda_{m}$ and denoted by $q_{m}\left(\lambda_{m}\right)$ since $\mu_{m}$ is irrelevant to the parking choices of the travlers and it is considered as a known parameter in the DCPM. In later discussion we let $q(\lambda)=\left\{q_{m}\left(\lambda_{m}\right)\right\}_{m=1}^{M} ; \lambda=\left\{\lambda_{m}\right\}_{m=1}^{M} ; \mu=\left\{\mu_{m}\right\}_{m=1}^{M}$.

Based on the problem description, this study seeks to establish the proposed DCPM by addressing the research challenges related to the participation and computation issues, respectively. First, we consider the uncertainty of the number of travelers in competing parking facilities, thus a Poisson game is introduced. Second, to promote the participations, we propose a mixed strategy game framework, which describes the parking competition incorporating drivers' parking choice preference. Namely, instead of telling the travelers exactly which parking facility to choose, the DCPM provides the vehicles a probability distribution among the parking facilities. An additional step could be taken to generate a specific facility according to the probability distribution and hence provide a deterministic guidance (a particular facility to choose) for the drivers/vehicles. For example, a random integer representing the recommended parking facility can be generated according to the probability distribution for each vehicle. Third, to ensure an online equilibrium parking decision can be efficiently reached among vehicles, we develop a distributed algorithm, which 
takes advantages of the distributed portable computational recourses. Below presents the technical details of our approaches.

4. Parking game

To establish a game framework for the proposed DCPM, the immediate challenge is the uncertainty of the population size in the parking coordination group. As we have seen above, the number of vehicles arriving to the $\mathrm{CBD}$ during a time interval can be considered as a random variable following Poisson distribution. Accordingly, we exploit Poisson games introduced in Myerson (1998) to handle this uncertainty. To facilitate the presentation of the proposed methodology, we briefly introduce Poisson game first.

\subsection{Poisson Game}

A Poisson game is a special case of game models with population uncertainty. It is defined by a fivetuple $(n, \Gamma, r, C, U)$. (i) $n$ represents the total number of players, which is a random variable obeying Poisson distribution with mean $\bar{n}$. (ii) $\Gamma$ represents the number of player types, indexed by $\kappa . \Gamma$ is a deterministic value. (iii) $r=\left\{r_{\kappa}\right\}_{\kappa=1}^{\Gamma}$ is a fixed probability distribution over the types. It determines the probability of each player belonging to type $\kappa$. Note that (i), (ii), and (iii) together implies that the number of players in each type $\kappa$ obeys Poisson distribution with the mean equal to $\bar{n} r_{\kappa}$. (iv) $A$ represents the number of all possible actions, indexed by $a$. The set of possible actions is the same for all the players and all the types. (v) Utility function $U(x, b, \kappa): \mathbb{N}^{A} \times\{1, \cdots, A\} \times\{1, \cdots, T\} \rightarrow \mathbb{R}$ gives the payoff of a player of type $\kappa$ taking action $b$ while the profile of action is $x=\left\{x_{a}\right\}_{a=1}^{A}$, where $x_{a}$ is a nonnegative integer indicating the number of players taking action $a . \mathbb{N}^{A}$ represents the set of all possible realizations of $x$, i.e., the set of nonnegative integer vectors of dimension $A$. An equilibrium of the Poisson game is a set of probability distributions, each corresponding to one type of players, over the action set, denoted by $p=$ $\left\{p_{a}^{\kappa}\right\}_{a=1, \cdots, A ; \kappa=1, \cdots, \Gamma}$ such that:

$$
\begin{gathered}
p_{a}^{\kappa}>0 \Rightarrow \bar{U}(a \mid \kappa, p)=\max _{b \in\{1, \cdots, A\}} \bar{U}(b \mid \kappa, p), \forall a=1, \cdots, A, \kappa=1, \cdots, \Gamma \\
p_{a}^{\kappa} \geq 0, \forall a=1, \cdots, A, \kappa=1, \cdots, \Gamma \\
\sum_{a=1}^{A} p_{a}^{\kappa}=1, \forall \kappa=1, \cdots, \Gamma,
\end{gathered}
$$

where $\bar{U}(b \mid \kappa, p)$ is the expected utility of a player of type $\kappa$ choosing action $b$, given the other players choose the actions according to the probability distributions in $p$. In fact, given $p$, according to the properties of Poisson distributions, the number of players choosing action $a$ also obeys a Poisson distribution with the mean being

$$
\sum_{\kappa=1}^{\Gamma}\left(\bar{n} r_{\kappa}\right) p_{a}^{\kappa} \triangleq \lambda_{a}
$$

Hence the expected utility function can be written as:

$$
\bar{U}(b \mid \kappa, p)=\sum_{x \in \mathbb{N}^{A}}\left[\prod_{a=1}^{A}\left(\frac{e^{-a}\left(\lambda_{a}\right)^{x_{a}}}{x_{a} !}\right)\right] U(x, b, \kappa) .
$$

Note that in Equation (5), the term in square brackets represents the probability that $x$ is the profile of action. If $U(x, b, \kappa)$ is a bounded function, it can be proven, by applying Kakutani fixed-point theorem (Myerson, 1998), that an equilibrium exists for this Poisson game.

\subsection{Parking Game with Uncertain Population}

Adopting the framework of Poisson game, this research develops a stochastic Poisson game (a Poisson game integrating stochastic user behavior) to model the resolution of the competition on parking facilities among the travelers in the parking coordination group, following the proposed DCPM. Three key elements to define the stochastic Poisson game are discussed below.

(i) Player Types $\{1,2, \cdots V\}$ : At each time interval $\tau$, vehicles/travelers searching for parking spots among 
the $M$ parking facilities in CBD are decomposed into $V$ different types, indexed by $v$, according to their destinations. Parking vehicles belonging to the same type $v$ share common preference on the parking facilities in the vicinity of the destinations. The number of players (travelers) in each type follows Poisson distribution with known mean $\bar{n}_{v}$ (the discussion to justify this assumption is given later). All parking vehicles, belonging to different types, together form the parking coordination group. Clearly, it is with uncertain number of players.

(ii) Actions $\{1,2, \cdots, M\}$ : The action set of travelers includes all the parking facilities in consideration. An action of a traveler can be understood as choosing one parking facility.

(iii) Expected Utility $\bar{U}(m \mid v, p)$ : Existing research shows that walking distance, parking cost and cruise opportunity are key factors to impact the parking decision of travelers (Lambe, 1996; Ellis, Rassam and Bennett, 1972; Asakura, Kashiwadani, 1994). Hence, this research considers the utility of the travelers as a weighted mixture of these three factors. More specifically, $\bar{U}(m \mid v, p)=\beta_{q}^{v} q_{m}+\beta_{s}^{v} s_{m}^{v}+\beta_{C}^{v} C_{m}^{v}$, where $q_{m}$ is defined in (2) representing the probability that the parking facility $m$ is fully occupied as it arrives; $s_{m}^{v}$ is the expected walking time from a parking facility to the destination. It is constant in this study. And $C_{m}^{v}$ is the parking cost, which is also constant in this study. Notice that among the three terms defining $\bar{U}(m \mid v, p)$, only $q_{m}$ is affected by the expected arrival rate $\lambda_{m}$, which is in turn determined by the parking decisions of all players as defined in Equation (1). Thus, we rewrite the expected utility as

$$
u_{m}^{v}(p) \triangleq \bar{U}(m \mid v, p)=\beta_{q}^{v} q_{m}\left(\lambda_{m}(p)\right)+\alpha_{m}^{v}
$$

where, $\alpha_{m}^{v} \triangleq \beta_{s}^{v} s_{m}^{v}+\beta_{C}^{v} C_{m}^{v}$ is the constant terms in the expected utility. Note that the travel time difference for the vehicles to drive to parking facilities is ignored as we assume that the travelers choose/decide parking facilities when they are in or close to the CBD. However, $\alpha_{m}^{v}$ can cover the factor without hurting the function of the proposed approach as travel time is treated as a constant value as $S_{m}^{v}$ and $C_{m}^{v}$.

Based on the MNL discrete model, the probability that traveler $v$ chooses parking lot $m$ is given by

$$
p_{m}^{v}=\frac{e^{-u_{m}^{v}}}{\sum_{m} e^{-u_{m}^{v}}}, m=1, \ldots, M
$$

Define function $\boldsymbol{p}(p)$ as

$$
\boldsymbol{p}(p)=\left\{\boldsymbol{p}_{\boldsymbol{m}}^{v}(p)\right\}_{m=1, \cdots, M ; v=1, \cdots, \mathrm{V}} ; \quad \boldsymbol{p}_{\boldsymbol{m}}^{v}(p)=\frac{e^{-u_{m}^{v}(p)}}{\sum_{m} e^{-u_{m}^{v}(p)}}, m=1, \cdots, M ; v=1, \cdots, \mathrm{V}
$$

The collective effect of the proposed parking game is captured by the equilibria of the stochastic Poisson game, which specifies $V$ number of probability distributions, $p^{*}=\left\{\left\{p_{m}^{*, \nu}\right\}_{m=1, \cdots, M}\right\}_{v=1, \cdots, V}$ such that

$$
\begin{gathered}
\boldsymbol{p}\left(p^{*}\right)=p^{*} \\
\sum_{m} p_{m}^{\nu *}=1, v=1, \cdots, \mathrm{V} \\
p_{m}^{v *} \geq 0, m=1, \cdots, M ; v=1, \cdots, \mathrm{V}
\end{gathered}
$$

The proposed stochastic Poisson game is different to Poisson game in two aspects. First, we notice that the equilibrium condition for Poisson game in Equation (3) states that for each type of the payers, only the actions that maximize the expected utility receive positive probabilities. This condition is analogy to the condition for the classical Wardrop equilibrium, which states that for each OD pair, the flow is only positive on the routes that minimize the travel cost. It is well known that by introducing behavior models, stochastic user equilibrium could be defined. Regarding to this research, by introducing travelers' parking behavior model (the multinomial logit discrete choice model), this research proposes the stochastic parking equilibrium conditions in Equation (8), which resembles those for stochastic user equilibrium. Second, as we can see that the expected utility function in Equation (5) is very difficult to evaluate in general. However, in the proposed parking problem, when we include $q_{m}$ as the key element in the utility function, the $M / M / c$ queue model provides us a closed-form formula for the expected utility. Therefore, we use $q_{m}$ in Equation (2) plus some extra constant terms as the expected utility function. 
Next, this study justifies the fit of using Poisson distribution to describe the size of parking vehicles in each type. First of all, Poisson distribution describes the probability of the occurrence of the number of events within a specific time period or a region of space. Thus, Poisson distribution is a good/reasonable fit to describe the number of parking vehicles arriving to a destination in CBD in each time interval, given the arrival of each individual parking vehicle is independent. This assumption has been used in literature such as (Yan, et al., 2011) and (Schlote et al., 2014). Second, the parking vehicles in a given type may include the remaining unallocated vehicles from the last time internal and new arriving parking vehicles in this time interval. We can still justify the fit of Poisson distribution by the decomposition law and superposition law of Poisson distribution (See Appendix D).

More specifically, parking vehicles in a type $v$ have the same priority $\left\{p_{m}^{v}\right\}$ to choose parking facilities $m=1, \ldots, M$ in the proposed stochastic Poisson game. Then, as each individual parking vehicle in type $v$ independently approaches to a parking facility $m$, it has a probability $q_{m}$ that the parking facility $m$ is full. Thus, the service of parking facilities in each time interval will decompose the parking vehicles in each type into two groups: (i) allocated with a probability $p$ and (ii) unallocated with a probability (1 $p)=\sum_{m=1}^{M} q_{m} p_{m}^{v}$. According to the decomposition law of Poisson distribution, the sizes of group (i) and group (ii) follows Poisson distribution, given that we assume parking vehicles arriving to a destination follows a Poisson process. Furthermore, we can use the similar argument to consider that the unallocated parking vehicles in group (ii) can be decomposed into another two groups: group (ii.a), in which the unallocated parking vehicles join the next round of DCPM, and group (ii.b), in which the unallocated parking vehicles change their trip plans and do not need parking service any more. Again, according to the decomposition law of Poisson distribution, the size of group (ii.a) follows Poisson distribution too. Therefore, the parking vehicles in a type $v$ in the next time interval may include new arriving parking vehicles to a destination and group (ii.a). Both of them follow Poisson distribution. According to the superposition law of Poisson distribution, the size of the parking vehicles in type $v$ in the next time interval still follows Poisson distribution. This dynamic process evolves over time. Therefore, we think Poisson distribution is a good fit to capture the size of parking vehicles in each type of players in the proposed stochastic Poisson game in a time interval.

Note that even though the parking vehicles in each type in a time interval may include unallocated parking vehicles in last time interval, the parking decision procedure following the proposed DCPM among different time intervals is independently processed. Namely, the DCPM forms a new PCG and coordinates their parking decisions in each time interval.

\subsection{Equivalent optimization model}

This study will prove that the proposed stochastic Poisson game is a potential game, i.e., its equilibrium is an optimal solution of an equivalent optimization model defined by Equations (9) to (12). Theorem 1 below presents this statement formally, followed by the proof.

$$
\begin{gathered}
(M P) \min \mathrm{Z}(p)=\sum_{m=1}^{M} \int_{0}^{\lambda_{m}} q_{m}(\omega) d \omega+\sum_{v=1}^{\mathrm{V}} \sum_{m=1}^{M} \frac{1}{\beta_{q}^{v}} p_{m}^{v} \ln \left(p_{m}^{v}\right) \\
+\sum_{v=1}^{\mathrm{V}} \sum_{m=1}^{M} \frac{\alpha_{m}^{v}}{\beta_{q}^{v}} p_{m}^{v} \\
\text { s.t. } \sum_{m=1}^{M} p_{m}^{v}=1, v=1, \ldots, \mathrm{V} \\
\lambda_{m}=\frac{1}{\Delta \tau} \sum_{v} \bar{n}_{v} p_{m}^{v}, m=1, \ldots, \mathrm{M} \\
p_{m}^{v} \geq 0, m=1, \ldots, M ; v=1, \ldots, \mathrm{V}
\end{gathered}
$$

Theorem 1: An optimal solution of the mathematical programming $(M P)$ is equivalent to the equilibrium parking probability distribution satisfying equilibrium conditions in Equation (8) for a parking 
coordination group with $V$ types of travelers competing for $M$ parking facility.

Proof: Notice that the objective function (9) in the MP is not defined at the points with $p_{m}^{v}=0$, for $m=$ $1, \ldots, M ; v=1, \ldots, \mathrm{V}$. Thus, to make the objective function continuous, this study lets $p_{m}^{v} \ln \left(p_{m}^{v}\right)=0$, given the fact that $\lim _{p_{m}^{v} \rightarrow 0} p_{m}^{v} \ln \left(p_{m}^{v}\right)=0$, for $\forall m=1, \ldots, M ; v=1, \ldots, \mathrm{V}$. The proof includes two steps.

Step I. This study first proves that for a local optimal solution, denoted by $p$, of the MP, it holds that $p_{m}^{v}>0, \forall m=1, \ldots, M ; v=1, \ldots, V$. Assume for the sake of contradiction that a local minimizer $p$ appears with $p_{m \prime}^{\nu \prime}=0$, for some $m^{\prime} \in\{1, \cdots, M\}$ and $v^{\prime} \in\{1, \cdots, \mathrm{V}\} ; m^{\prime \prime} \neq m^{\prime}$ such that $p_{m \prime \prime}^{v^{\prime \prime}}=x>0$, given $\sum_{m} p_{m}^{v \prime}=1$. Then, for a small value of $\varepsilon>0$, another solution $\bar{p}$ is defined below:

Therefore,

$$
\begin{gathered}
\bar{p}_{m{ }^{\prime \prime}}^{\nu \prime}=p_{m{ }^{\prime}}^{v \prime}-\varepsilon=x-\varepsilon, \\
\bar{p}_{m \prime}^{\nu \prime}=p_{m \prime}^{\nu \prime}+\varepsilon=\varepsilon, \\
\bar{p}_{m}^{v \prime}=p_{m}^{v \prime}, \text { for any other } m \neq m^{\prime} \text { or } m^{\prime \prime}, v=v^{\prime} .
\end{gathered}
$$

Then we compare the values of $\mathrm{Z}(\bar{p})$ and $\mathrm{Z}(p)$.

$$
\begin{aligned}
& \mathrm{Z}(\bar{p})-\mathrm{Z}(p)=\int_{0}^{\bar{\lambda}_{m \prime}} q_{m}(\omega) d \omega-\int_{0}^{\lambda_{m^{\prime}}} q_{m}(\omega) d \omega+\int_{0}^{\bar{\lambda}_{m^{\prime \prime}}} q_{m}(\omega) d \omega-\int_{0}^{\lambda_{m^{\prime \prime}}} q_{m}(\omega) d \omega \\
& +\frac{1}{\beta_{q}^{v^{\prime}}} \bar{p}_{m^{\prime}}^{v^{\prime}} \ln \left(\bar{p}_{m^{\prime}}^{v^{\prime}}\right)-\frac{1}{\beta_{q}^{v^{\prime}}} p_{m^{\prime}}^{v^{\prime}} \ln \left(p_{m^{\prime}}^{v^{\prime}}\right)+\frac{1}{\beta_{q}^{v^{\prime}}} \bar{p}_{m^{\prime \prime}}^{v^{\prime}} \ln \left(\bar{p}_{m^{\prime \prime}}^{v^{\prime}}\right)-\frac{1}{\beta_{q}^{v^{\prime}}} p_{m^{\prime \prime}}^{v^{\prime}} \ln \left(p_{m^{\prime \prime}}^{v^{\prime}}\right) \\
& +\frac{\alpha_{m^{\prime}}^{v^{\prime}}}{\beta_{q}^{v^{\prime}}} \bar{p}_{m^{\prime}}^{v^{\prime}}-\frac{\alpha_{m^{\prime}}^{v^{\prime}}}{\beta_{q}^{v^{\prime}}} p_{m^{\prime}}^{v^{\prime}}+\frac{\alpha_{m^{\prime \prime}}^{v^{\prime}}}{\beta_{q}^{v^{\prime}}} \bar{p}_{m^{\prime \prime}}^{v^{\prime}}-\frac{\alpha_{m^{\prime \prime}}^{v^{\prime}}}{\beta_{q}^{v^{\prime}}} p_{m^{\prime \prime}}^{v^{\prime}} \\
& =\underbrace{\left(\int_{\lambda_{m^{\prime}}}^{\lambda_{m^{\prime}}+\frac{\varepsilon}{\Delta \tau}} q_{m}(\omega) d \omega+\int_{\lambda_{m^{\prime \prime}-\frac{\varepsilon}{\Delta \tau}}}^{\lambda_{m^{\prime \prime}}} q_{m}(\omega) d \omega\right)}_{\Delta Z 1} \\
& +\underbrace{\left(\frac{1}{\beta_{q}^{v^{\prime}}}(\varepsilon \ln \varepsilon+(x-\varepsilon) \ln (x-\varepsilon)-x \ln x)\right)}_{\Delta Z 2}+\underbrace{\left(\frac{\alpha_{m^{\prime}}^{v^{\prime}}}{\beta_{q}^{v^{\prime}}}-\frac{\alpha_{m^{\nu^{\prime \prime}}}^{v^{\prime}}}{\beta_{q}^{v^{\prime}}}\right) \varepsilon}_{\Delta Z 3}
\end{aligned}
$$

Denote the three parts of Equation (19) as $\Delta Z 1, \Delta Z 2$, and $\Delta Z 3$. Then since $q_{m}(\omega) \leq 1$, we must have

$$
\Delta Z 1 \leq \frac{\varepsilon}{\Delta \tau}+\frac{\varepsilon}{\Delta \tau}=\frac{2}{\Delta \tau} \varepsilon
$$

Also,

$$
\Delta Z 3 \leq\left|\frac{\alpha_{m^{\prime}}^{v^{\prime}}}{\beta_{q}^{v^{\prime}}}-\frac{\alpha_{m^{\prime \prime}}^{v^{\prime}}}{\beta_{q}^{v^{\prime}}}\right| \varepsilon
$$


Without loss of generality, assume that $\varepsilon<\frac{x}{2}$. Hence $x-\varepsilon \in\left[\frac{x}{2}, x\right]$. Since $x \ln x$ is differentiable on $\left[\frac{x}{2}, x\right]$, by mean value theorem, we must have

$$
(x-\varepsilon) \ln (x-\varepsilon)-x \ln x=(1+\ln y) \varepsilon,
$$

where $y \in[x-\varepsilon, x] \subseteq\left[\frac{x}{2}, x\right]$. Since $\ln y$ is a continuous function on $\left[\frac{x}{2}, x\right]$, there must exist a real number $B$ such that $\ln y \leq B$ for all $y \in\left[\frac{x}{2}, x\right]$. Therefore,

$$
(x-\varepsilon) \ln (x-\varepsilon)-x \ln x \leq(1+B) \varepsilon .
$$

Overall, we have:

$$
\mathrm{Z}(\bar{p})-\mathrm{Z}(p) \leq\left(\frac{2}{\Delta \tau}+\left|\frac{\alpha_{m^{\prime}}^{v^{\prime}}}{\beta_{q}^{v^{\prime}}}-\frac{\alpha_{m^{\prime \prime}}^{v^{\prime}}}{\beta_{q}^{v^{\prime}}}\right|+1+B+\frac{1}{\beta_{q}^{v^{\prime}}} \ln \varepsilon\right) \varepsilon
$$

Let

$$
B^{\prime}=\frac{2}{\Delta \tau}+\left|\frac{\alpha_{m^{\prime}}^{v^{\prime}}}{\beta_{q}^{v^{\prime}}}-\frac{\alpha_{m^{\prime \prime}}^{v^{\prime}}}{\beta_{q}^{v^{\prime}}}\right|+1+B
$$

Take $\varepsilon<\min \left(\frac{x}{2}, e^{-B^{\prime} \beta_{q}^{v^{\prime}}}\right)$, we can see that

$$
\mathrm{Z}(\bar{p})-\mathrm{Z}(p)<0
$$

This conflicts with our assumption that $p$ is a local minimizer. This concludes the proof of the first step.

Step II. Given the conclusion above, this study next proves that equivalence stated in Theorem 1 . The idea is to demonstrate that the Karush-Kuhn-Tucker (KKT) condition of the MP is equivalent to the equilibrium in Equation (7). The KKT condition of the MP is given below.

$$
\begin{gathered}
p_{m}^{v}\left(q_{m}\left(\frac{1}{\Delta \tau} \sum_{v} \bar{n}_{v} p_{m}^{v}\right)+\frac{1}{\beta_{q}^{v}}\left(\ln \left(p_{m}^{v}\right)+1\right)+\frac{\alpha_{m}^{v}}{\beta_{q}^{v}}-\pi^{v}\right)=0, m=1, \ldots, M ; v=1, \ldots, V \\
\sum_{m=1}^{M} p_{m}^{v}=1, v=1, \ldots, V \\
q_{m}\left(\frac{1}{\Delta \tau} \sum_{v} \bar{n}_{v} p_{m}^{v}\right)+\frac{1}{\beta_{q}^{v}}\left(\ln \left(p_{m}^{v}\right)+1\right)+\frac{\alpha_{m}^{v}}{\beta_{q}^{v}}-\pi^{v} \geq 0 \\
p_{m}^{v} \geq 0
\end{gathered}
$$

Where, $\pi^{v}$ is the multiplier of the constraint (10). Given the proof in step I, we obtain that $p_{m}^{v}>0$ for any local optimal solution. Then Equation (22) indicates that

$$
q_{m}\left(\frac{1}{\Delta \tau} \sum_{v} \bar{n}_{v} p_{m}^{v}\right)+\frac{1}{\beta_{q}^{v}}\left(\ln \left(p_{m}^{v}\right)+1\right)+\frac{\alpha_{m}^{v}}{\beta_{q}^{v}}=\pi^{v}
$$

Note that here $q_{m}\left(\sum_{v} \bar{n}_{v} p_{m}^{v}\right)$ gives the value of $q_{m}$ in utility function in Equation (6). Then, rearrange Equation (26), we found that

$$
p_{m}^{v}=e^{\beta_{q}^{v} \pi^{v}-1} e^{-\left(\alpha_{m}^{v}+\beta_{q}^{v} q_{m}\right)}=e^{\beta_{q}^{v} \pi^{v}-1} e^{-u_{m}^{v}}=\Upsilon e^{-u_{m}^{v}}
$$

Combining Equation (23), we obtain the constant value of $\Upsilon=\frac{1}{\sum_{m} e^{-u_{m}^{v}}}$, and then we know that

$$
p_{m}^{v}=\frac{e^{-u_{m}^{v}}}{\sum_{m} e^{-u_{m}^{v}}}, m=1, \ldots, M ; v=1, \ldots, V
$$

Thus Equation (28) implies the equilibrium condition given in Equation (8). From the other side, it is easy to show the parking equilibrium assignment will satisfy the optimal conditions of the MP from Equations (22) to (25). This completes the proof for Theorem 1. 


\section{Distributed Algorithm}

A simultaneously updating distributed algorithm (SDA) is used to search for an equilibrium solution of the DCPM to ensure the scalability of the solution method. Recall the features of stochastic Poisson game: the same type of travelers in the parking coordination group has the same priority to the alternatives in the action set (parking facilities). This study proposes a distributed algorithm, in which each type of players (say those with the same/similar destinations) randomly picks a "spokesman" representing all other members of this type to negotiate the parking preference with "spokesmen" from other types of players. Then the proposed parking game with uncertain number of players is converted to a mixed strategic game with fixed number of players (i.e. spokesmen), which tries to maximum the utility of the type he represents. To facilitate the articulation, the mixed strategy game formed by the spokesmen is referred as to the MGS hereafter.

The procedure of the distributed algorithm is as below, in which $\wp_{\mathrm{m}}^{\mathrm{v}}(\mathrm{t})$ presents the probability that vehicle $v$ chooses parking facility $m$ at iteration $t ; \mathrm{p}_{\mathrm{m}}^{v}(\mathrm{t}+1)$ represents the target probability that vehicle $v$ chooses parking facility $m$ at iteration $t+1 ; \mathrm{p}_{\mathrm{m}}^{v}(\mathrm{t}+1)$ is calculated by Equation $(7) ; d_{m}^{v}=\mathrm{p}_{\mathrm{m}}^{v}(\mathrm{t}+$ 1) $-\wp_{\mathrm{m}}^{v}(\mathrm{t})$ represents the moving direction and $\gamma$ represents the moving step size to sustain the convergence of the distributed algorithm.

1. Initialization: $q_{m}, \mu_{m}, C_{m}, S_{m}^{v}, p_{m}^{v}$

2. For $t=1,2, \ldots$

If the convergence criterion is satisfied, exit

Otherwise

(1) Update $\lambda_{m}(t), m=1, \ldots, M$ according to Equation (1)

(2) Update $q_{m}(t), m=1, \ldots, M$ according to Equation (2)

(3) Update $\left\{\wp_{m}^{v}(t)\right\}_{m=1}^{M}$ for all types travelers $v=1, \ldots, V$ by

$$
\begin{gathered}
\wp_{m}^{v}(t+1)=\wp_{m}^{v}(t)+\gamma(t) d_{m}^{v} \\
d_{m}^{v}=\left(p_{m}^{v}(t+1)-\wp_{m}^{v}(t)\right)
\end{gathered}
$$

End

Specially, Step 1 starts the algorithm by assigning initial input values: $q_{m}, \mu_{m}, C_{m}, S_{m}^{v}, p_{m}^{v}$. Step 2 iteratively searches the equilibrium of the proposed game. More exactly, the algorithm first checks if $\left\{\mathrm{p}_{\mathrm{m}}^{v}\right\}$ satisfies the convergence criterion. If yes, the algorithm terminates. Otherwise, according to current parking priority of all types of vehicles, the algorithm of Step 2 (1) updates the arrival rate $\left\{\lambda_{\mathrm{m}}\right\}$ at each parking facility $\mathrm{m}$; next, according to $\left\{\lambda_{\mathrm{m}}\right\}$, Step 2 (2) updates $\left\{\mathrm{q}_{\mathrm{m}}\right\}$, the probability that facility $\mathrm{m}$ is full; last, according to $\left\{\mathrm{q}_{\mathrm{m}}\right\}$, Step 2 (3) updates the parking priority of each type of vehicles by Equation (29). After that, the algorithm goes back to the beginning of Step 2. These processes in Step 2 repeat until the convergence criterion is satisfied. Furthermore, this study proves the convergence of the algorithms and provides the formulations for the step size $\gamma$ of the algorithm in the following section.

\subsection{Convergence of the SDA}

This section seeks to prove the convergence of the SDA using Theorem 2 below, which is given in Bazaraa et al. (2006)) for the convergence of iterative algorithms.

Theorem 2: (Bazaraa et al. (2006)). Let $X$ be a non-empty closed set in $R^{n}$, and let the non-empty set $S \subseteq$ $X$ be the solution set. Let $A: X \rightarrow X$ be a point-to-set map. Given $x_{0} \in X$, the sequence $\left\{x_{k}\right\}$ is generated iteratively as follows: if $x_{k} \in S$ then stop; otherwise, let $x_{k+1} \in A\left(x_{k}\right)$, replace $k$ by $k+1$, and repeat. Suppose the sequence $x_{1}, x_{2}, \ldots$, produced by the algorithm is in a compact set $X$, and suppose that there exists a continuous function $\varphi$, called the descent function, such that $\varphi(y)<\varphi(x)$ if $x \notin S$ and $y \in A(x)$. If the map $A$ is closed over the complement of $S$, then either the algorithm stops in a finite number of steps with a point in $S$ or it generates the infinite sequence $\left\{x_{k}\right\}$ such that:

(1) every convergent subsequence of $\left\{x_{k}\right\}$ has a limit in $S$, i.e., all accumulation point of $\left\{x_{k}\right\}$ belongs to $S$;

(2) $\varphi\left(x_{k}\right) \rightarrow \varphi(x)$ for some $x \in S$. 
According to Theorem 2, the proof of the convergence of the SDA includes the three key parts and the technical details to prove them are given in the following sections.

1) Uniqueness of the equilibrium resolution among spokesmen

2) Existences a descent function $\varphi$ for the SDA.

3) Closeness of the map from $\wp(t)=\left\{\wp_{m}^{v}(t)\right\}$ to $\wp(t+1)=\left\{\wp_{m}^{v}(t+1)\right\}$.

5.1.1.Transformation of $q$ function

Before presenting the proof of the convergence of the SDA, this research noticed that $q(\lambda)$ in Equation (2) is a piece-wise function (non-differentiable at $\rho=1$ ). It brings difficulty to the convergence of the algorithm. This study thus considers using an approximation function to substitute Equation (2) in the algorithm. To explore the proper approximation function, this study exams the curvature feature of Equation (2), which are demonstrated by the first and second order derivatives of Equation (2) as $\rho<1$. For simplicity of notation we remove the subscript $m$ in this section. It is noticed that $q(\lambda)$ is a separable function of $\lambda$, given $\mu$ is considered as constant since it is not affected by the DCPM. As $\rho=\frac{\lambda}{\mu}$, it suffices to examine $q^{\prime}(\rho)$ to explore the features of $q^{\prime}(\lambda)$. We have $q^{\prime}(\rho)$ :

$$
q^{\prime}(\rho)=\left(\frac{A(\rho)}{B(\rho)+A(\rho)}\right)^{\prime}=\frac{A^{\prime}(\rho)}{B(\rho)+A(\rho)}-\frac{A(\rho)\left[B^{\prime}(\rho)+A^{\prime}(\rho)\right]}{[B(\rho)+A(\rho)]^{2}}=\frac{A^{\prime}(\rho) B(\rho)-A(\rho) B^{\prime}(\rho)}{[B(\rho)+A(\rho)]^{2}}
$$

Where,

$$
\begin{gathered}
A(\rho)=\frac{\rho^{c}}{(1-\rho)}, B(\rho)=\frac{c !}{c^{c}} \sum_{k=0}^{c-1} \frac{(c \rho)^{k}}{k !} \\
A^{\prime}(\rho)=\frac{\rho^{c-1}[c(1-\rho)+\rho]}{(1-\rho)^{2}}, \text { and } B^{\prime}(\rho)=\frac{c !}{c^{c-1}} \sum_{k=0}^{c-2} \frac{(c \rho)^{k}}{k !} .
\end{gathered}
$$

Apparently, the denominator of $q^{\prime}(\rho)$ is positive, we next exam the numerator of $q^{\prime}(\rho)$.

$$
\begin{aligned}
& A^{\prime}(\rho) B(\rho)-A(\rho) B^{\prime}(\rho) \\
& =\frac{\rho^{c-1}[c(1-\rho)+\rho]}{(1-\rho)^{2}} \frac{c !}{c^{c}} \sum_{k=0}^{c-1} \frac{(c \rho)^{k}}{k !}-\frac{\rho^{c}}{(1-\rho)} \frac{c !}{c^{c-1}} \sum_{k=0}^{c-2} \frac{(c \rho)^{k}}{k !} \\
& =\frac{c !}{c^{c}} \frac{\rho^{c-1}}{(1-\rho)^{2}}\left\{[c(1-\rho)+\rho] \sum_{k=0}^{c-1} \frac{(c \rho)^{k}}{k !}-c \rho(1-\rho) \sum_{k=0}^{c-2} \frac{(c \rho)^{k}}{k !}\right\} \\
& =\frac{c !}{c^{c-1}} \frac{\rho^{c-1}}{(1-\rho)^{2}}\left\{\sum_{k=0}^{c-2} \frac{(c \rho)^{k}}{k !}\left[c(1-\rho)^{2}+\rho\right]+\frac{(c \rho)^{c-1}}{(c-1) !}[c(1-\rho)+\rho]\right\}>0, \text { for } \rho<1
\end{aligned}
$$

According to Equations (30) and (32), it is clear that $q^{\prime}(\rho)>0$ when $\rho<1$, i.e., $q^{\prime}(\lambda)>0$ for $\lambda<\mu$. Therefore, $q(\lambda)$ is an increasing function respect to $\lambda$, when $\lambda<\mu$. We next exam the second derivative of $Q(\lambda)$ respect to $\rho$, as $\rho<1$.

where

$$
q^{\prime \prime}(\rho)=\underbrace{\frac{A^{\prime \prime}(\rho) B(\rho)-A(\rho) B^{\prime \prime}(\rho)}{[B(\rho)+A(\rho)]^{2}}}_{q I}+\underbrace{\frac{2\left\{\left[A^{\prime}(\rho)+B^{\prime}(\rho)\right]\left[A^{\prime}(\rho) B(\rho)-A(\rho) B^{\prime}(\rho)\right]\right\}}{[B(\rho)+A(\rho)]^{3}}}_{q I I}
$$

$$
A^{\prime \prime}(\rho)=\frac{\rho^{c-2}\left[c(c-1)(1-\rho)^{2}+2 c \rho(1-\rho)+2 \rho^{2}\right]}{(1-\rho)^{3}}, B^{\prime \prime}(\rho)=\frac{c !}{c^{c-2}} \sum_{k=0}^{c-3} \frac{(c \rho)^{k}}{k !}
$$

According to Equations (31) and (32), it is noticed that $A^{\prime}(\rho)>0, B^{\prime}(\rho)>0$, and $A^{\prime}(\rho) B(\rho)-$ $A(\rho) B^{\prime}(\rho)>0$ in Equation (33) when $\rho<1$. Thus, part II in Equation (33) is positive for $\rho<1$. We next consider the numerator of part I in $q^{\prime \prime}(\rho)$. When $c=1,2, B^{\prime \prime}(\rho)=0$, thus $q I>0$. For $c \geq 3$, the analysis is given below. 


$$
\begin{aligned}
& A^{\prime \prime}(\rho) B(\rho)-A(\rho) B^{\prime \prime}(\rho) \\
& =\frac{\rho^{c-2}\left[c(c-1)(1-\rho)^{2}+2 c \rho(1-\rho)+2 \rho^{2}\right]}{(1-\rho)^{3}} \frac{c !}{c^{c}} \sum_{k=0}^{c-1} \frac{(c \rho)^{k}}{k !}-\frac{\rho^{c}}{(1-\rho)} \frac{c !}{c^{c-2}} \sum_{k=0}^{c-3} \frac{(c \rho)^{k}}{k !} \\
& =\frac{c !}{c^{c}} \frac{\rho^{c-2}}{(1-\rho)^{3}}\{\sum_{k=0}^{c-3} \frac{(c \rho)^{k}}{k !} \underbrace{\left[c(c-1)(1-\rho)^{2}+2 c \rho(1-\rho)+2 \rho^{2}-c^{2} \rho^{2}(1-\rho)^{2}\right]}_{\Delta 1} \\
& +\underbrace{\left[\frac{(c \rho)^{c-2}}{(c-2) !}+\frac{(c \rho)^{c-1}}{(c-1) !}\right]\left[c(c-1)(1-\rho)^{2}+2 c \rho(1-\rho)+2 \rho^{2}\right]}_{\Delta 2}\}
\end{aligned}
$$

Clearly, $\Delta 2$ in Equation (35) is positive for $\rho<1$. To identify the sign of Equation (35), we only need to consider the part of $\Delta 1$, which can be transformed by the following process.

$$
\begin{aligned}
& \Delta 1=c(c-1)(1-\rho)^{2}+2 c \rho(1-\rho)+2 \rho^{2}-c^{2} \rho^{2}(1-\rho)^{2} \\
& =c\left[(c-1)+(4-2 c) \rho-3 \rho^{2}+2 c \rho^{3}-c \rho^{4}\right]+2 \rho^{2} \\
& \geq\left[(c-1)+(4-2 c) \rho-3 \rho^{2}+2 c \rho^{3}-c \rho^{4}\right] \triangleq f(\rho)
\end{aligned}
$$

It is noticed that

$$
f(\rho)=(c-1)+(4-2 c) \rho-3 \rho^{2}+2 c \rho^{3}-c \rho^{4}
$$

and

$$
\begin{gathered}
f^{\prime}(\rho)=(4-2 c)-6 \rho+6 c \rho^{2}-4 c \rho^{3} \\
f^{\prime \prime}(\rho)=6\left(1+2 c \rho-2 c \rho^{2}\right)
\end{gathered}
$$

Letting $f^{\prime \prime}(\rho)=0$, we can obtain two stationary point of $f^{\prime}(\rho): \rho_{1}=\frac{c-\sqrt{c^{2}+2 c}}{2 c}<0$ and $\rho_{2}=\frac{c+\sqrt{c^{2}+2 c}}{2 c}>$ 1. Because $\rho \in[0,1)$, this region is within $\left[\rho_{1}, \rho_{2}\right]$. So $f^{\prime}(\rho)$ is monotonic as $\rho \in[0,1)$. $f^{\prime}(0)=4-$ $2 c<0$ and $f^{\prime}(1)=-2<0$. So, we claim $f^{\prime}(\rho)<0$ as $\rho \in[0,1)$. Hence, $f(\rho)$ is monotonically decreasing in $\rho \in[0,1)$. Given $f(1)=0$, we have $f(\rho) \geq 0$ in $\rho \in[0,1)$. Then, we obtain that $\Delta 1>0$ in Equation (35). Combing the discussions for the first and second elements in Equation (33), we conclude that $q^{\prime \prime}(\rho)>0$ when $\rho<1$

According to the curvature characteristics of the $q(\rho)$ function demonstrate by $q^{\prime}(\rho)$ and $q^{\prime \prime}(\rho)$, we can predict that both $q(\rho)$ and its slope monotonically increase with the increase of $\rho$; but, $q(\rho)=1$ suddenly at $\rho=1$ (i.e., the slope of $q(\rho)$ becomes zero suddenly at $\rho=1$ ). It is noticed that the slope of a sigmoid functions display a similar trend of first increasing and then decreasing to almost zero, but in a smooth fashion. Therefore, this study uses a sigmoid function such as Equation (39) below to approximate $q(\lambda)$ so that we can smoothen the sudden change of the slope of $q(\rho)$ at $\rho=1$.

$$
\hat{q}(\lambda)=\frac{1}{1+b e^{a \lambda}}, a<0
$$

where $a$ and $b$ are parameters. It is noticed that $\hat{q}^{\prime}(\lambda)>0$ as $a<0$. It is true this smoothening will introduce an error but it will help in the distributed algorithm design and convergence discussion. Moreover, the shape of sigmoid function is adjustable by selecting their parameters $a$ and $b$. To ensure an acceptable difference between $\hat{q}(\lambda)$ and $q(\lambda)$, this study uses regression methods to find the best fit. We obtain a regression model with R-square equal to 0.8118 on the average in our experimental study (see Appendix A). Note that this smoothening process does not have to use sigmoid functions in the proposed methodology. It can be any continuous, first and second order differentiable function, if its slop demonstrates the trend of first increasing and then decreasing to near zero.

5.1.2.Uniqueness of the equilibrium

Next, we demonstrate the uniqueness of the equilibrium for the MGS, using $\hat{q}_{m}\left(\lambda_{m}\right)$ to approximiate the function $q_{m}\left(\lambda_{m}\right)$ for all $m=1, \cdots, M$. For the simplicity of notation, we ignore the hat on the functions. The formal statement on uniqueness is given in Lemma 1, followed by its proof.

Lemma 1: The Mixed Strategy Game formed by the Spokesmen has a unique equilibrium. 
Proof: According to Theorem 1, if the MP in Equations (9)-(12) has unique optimal solution, we can claim that the MGS has a unique equilibrium. Below presents the technique details for the proof.

It is clear that the MP has a convex feasible region formed by linear constraints. This study next shows that the objective function in Equation (9) is strictly convex. The Hessian matrix $H_{M \Gamma \times M \Gamma}$ of Equation (9) is of a format with two parts as below.

$$
\begin{gathered}
H=\left[\begin{array}{ccc}
h_{1}{ }^{\prime} & \cdots & \\
\vdots & h_{m}{ }^{\prime} & \vdots \\
\cdots & h_{M}{ }^{\prime}
\end{array}\right]_{M V \times M V}+\left[\begin{array}{ccc}
\frac{1}{\beta_{q}^{1} p_{1}^{1}} & \cdots & \\
\vdots & \ddots & \vdots \\
& \cdots & \frac{1}{\beta_{q}^{V} p_{M}^{V}}
\end{array}\right]_{M V \times M V} \\
h_{m}^{\prime}=\left[\begin{array}{ccc}
q_{m}^{\prime}\left(\frac{\bar{n}_{1}}{\Delta \tau}\right)^{2} & \cdots & q_{m}^{\prime}\left(\frac{\bar{n}_{1} \bar{n}_{V}}{\Delta \tau^{2}}\right) \\
\vdots & \ddots & \vdots \\
q_{m}^{\prime}\left(\frac{\bar{n}_{V} \bar{n}_{1}}{\Delta \tau^{2}}\right) & \cdots & q_{m}^{\prime}\left(\frac{\bar{n}_{V}}{\Delta \tau}\right)^{2}
\end{array}\right]_{V \times V}=\frac{q_{m}^{\prime}}{(\Delta \tau)^{2}}\left[\begin{array}{ccc}
\left(\bar{n}_{1}\right)^{2} & \cdots & \left(\bar{n}_{1} \bar{n}_{V}\right) \\
\vdots & \ddots & \vdots \\
\left(\bar{n}_{V} \bar{n}_{1}\right) & \cdots & \left(\bar{n}_{V}\right)^{2}
\end{array}\right]_{V \times V}=\frac{q_{m}^{\prime}}{(\Delta \tau)^{2}} n n^{T}
\end{gathered}
$$

where, $q_{m}^{\prime}=\frac{-a b e^{a \lambda_{m}}}{1+b e^{a \lambda_{m}}}>0$ since $a<0$ according to Equation (39); $n=\left[\bar{n}_{1}, \ldots, \bar{n}_{V}\right]^{T}$. It can be shown that $h_{m}^{\prime}$ is positive semi-definite since for any vector $\nu$.

$$
v^{T} n n^{T} v=\left(v^{T} n\right)\left(v^{T} n\right)^{T}=\left(\sum_{i=1}^{\mathrm{V}} \bar{n}_{i} x_{i}\right)^{2} \geq 0, \forall v \in \mathbb{R}^{V}
$$

Consequently, we state that the Hessian matrix $H$ is positive definite and the objective function of MP is strictly convex. According to optimization theory, this study concludes Lemma 1.

\subsubsection{Existence of a descent function}

To present the existence of a descent function for the SDA, this study uses the objective function of the equivalent MP as the potential function. Then we check the sign of $\mathrm{Z}(\wp(t+1))-\mathrm{Z}(\wp(t))$. Recall that $\wp_{m}^{v}(t)$ represents the probability that vehicle type $v$ takes parking facility $m$ at iteration $t$. It is calculated by Equation (29) and $\wp(t)=\left\{\wp_{m}^{v}(t)\right\}$. $p_{m}^{v}(t)$ represents the target probability vehicle type $v$ takes parking facility $m$ at iteration $t$. It is calculated by Equation (7) and $p(t)=\left\{p_{m}^{v}(t)\right\}$. Using Taylor expansion of $Z(\wp(t+1))$ at $(\wp(t))$, we have

$$
\begin{aligned}
& Z(\wp(t+1))-Z(\wp(t)) \\
& =(\wp(t+1)-\wp(t))^{T} Z^{\prime}(\wp(t))+\frac{1}{2}(\wp(t+1)-\wp(t))^{T} H(\widetilde{\wp}(t))(\wp(t+1)-\wp(t)) \\
& =\gamma(t) d^{T} Z^{\prime}(\wp(t))+\gamma(t)^{2} \frac{1}{2} d^{T} H(\widetilde{\wp}(t)) d
\end{aligned}
$$

where, $\wp(t+1)-\wp(t)=\gamma d=\gamma(p(t+1)-\wp(t))$; $\widetilde{\wp}(t)$ is between $\wp(t+1)$ and $\wp(t)$. Moreover, we have the discussion about $d^{T} Z^{\prime}(\wp(t))$ below. 


$$
\begin{aligned}
& d^{T} Z^{\prime}(\wp(t)) \\
& =\sum_{m=1}^{M} \sum_{v=1}^{V}\left(p_{m}^{v}(t+1)-\wp_{m}^{v}(t)\right)\left(q_{m}\left(\lambda_{m}(t)\right)+\frac{1}{\beta_{q}^{v}}\left(\ln \left(\wp_{m}^{v}(t)\right)+1\right)+\frac{\alpha_{m}^{v}}{\beta_{q}^{v}}\right) \\
& =\sum_{m=1}^{M} \sum_{v=1}^{V}\left(p_{m}^{v}(t+1) q_{m}\left(\lambda_{m}(t)\right)+\frac{1}{\beta_{q}^{v}} p_{m}^{v}(t+1)\left(\ln \left(p_{m}^{v}(t+1)\right)+\alpha_{m}^{v}\right)\right) \\
& -\left(\wp_{m}^{v}(t) q_{m}\left(\lambda_{m}(t)\right)+\frac{1}{\beta_{q}^{v}} \wp_{m}^{v}(t)\left(\ln \left(\wp_{m}^{v}(t)\right)+\alpha_{m}^{v}\right)\right)+\frac{1}{\beta_{q}^{v}} p_{m}^{v}(t+1)\left(\ln \frac{\wp_{m}^{v}(t)}{p_{m}^{v}(t+1)}\right)
\end{aligned}
$$

According to Equation (28) in (Du et al, 2014) (see Appendix B for details), we have,

$$
\begin{gathered}
\left(p_{m}^{v}(t+1) q_{m}\left(\lambda_{m}(t)\right)+\frac{1}{\beta_{q}^{v}} p_{m}^{v}(t+1)\left(\ln \left(p_{m}^{v}(t+1)\right)+\alpha_{m}^{v}\right)\right) \\
-\left(\wp_{m}^{v}(t) q_{m}\left(\lambda_{m}(t)\right)+\frac{1}{\beta_{q}^{v}} \wp_{m}^{v}(t)\left(\ln \left(\wp_{m}^{v}(t)\right)+\alpha_{m}^{v}\right)\right) \leq 0
\end{gathered}
$$

Then,

$$
d^{T} Z^{\prime}(\wp(t)) \leq-\sum_{m=1}^{M} \sum_{v=1}^{V} \frac{1}{\beta_{q}^{v}} p_{m}^{v}(t+1)\left(\ln \frac{p_{m}^{v}(t+1)}{\wp_{m}^{v}(t)}\right)
$$

According to the inequality stated in Theorem 11 given by Borwein and Lewis (2006) (see Appendix C for details), we have

$$
\begin{aligned}
& d^{T} Z^{\prime}(\wp(t)) \leq \sum_{v=1}^{V}-\frac{1}{\beta_{q}^{v}} \sum_{m=1}^{M} p_{m}^{v}(t+1)\left(\ln \frac{p_{m}^{v}(t+1)}{\wp_{m}^{v}(t)}\right) \\
& \leq-\sum_{v=1}^{V} \frac{1}{2 \beta_{q}^{v}}\left(\sum_{m=1}^{\mathrm{M}}\left|p_{m}^{v}(t+1)-\wp_{m}^{v}(t)\right|\right)^{2} \\
& \leq-\sum_{v=1}^{V} \frac{1}{2 \beta_{q}^{v}} \sum_{m=1}^{\mathrm{M}}\left(p_{m}^{v}(t+1)-\wp_{m}^{v}(t)\right)^{2}
\end{aligned}
$$

Thus, we have the upper bound for $Z(\wp(t+1))-Z(\wp(t))$ below.

$$
\begin{aligned}
& Z(\wp(t+1))-Z(\wp(t)) \\
& \leq-\gamma(t) \sum_{v=1}^{V} \frac{1}{2 \beta_{q}^{v}} \sum_{m=1}^{M}\left(p_{m}^{v}(t+1)-\wp_{m}^{v}(t)\right)^{2}+\gamma(t)^{2} \frac{1}{2} d^{T} H(\widetilde{\wp}(t)) d
\end{aligned}
$$

To make sure $Z(\wp(t+1))-Z(\wp(t))<0$, we need:

$$
\begin{aligned}
& -\gamma(t) \sum_{v=1}^{V} \frac{1}{2 \beta_{q}^{v}} \sum_{m=1}^{M}\left(p_{m}^{v}(t+1)-\wp_{m}^{v}(t)\right)^{2}+\gamma(t)^{2} \frac{1}{2} d^{T} H(\widetilde{\wp}(t)) d<0 \\
& \Leftrightarrow \gamma(t)<\frac{\sum_{v=1}^{V} \frac{1}{2 \beta_{q}^{v}} \sum_{m=1}^{M}\left(p_{m}^{v}(t+1)-\wp_{m}^{v}(t)\right)^{2}}{\frac{1}{2} d^{T} H(\widetilde{\wp}(t)) d} \triangleq \gamma_{B}(t)
\end{aligned}
$$

We next give detailed discussion on the value of $\gamma_{B}(t)$. It is noticed that the element $\widetilde{\wp}(t)$ is between $\wp(t+1)$ and $\wp(t)$. Thus, $\gamma_{B}(t)$ is related to the parking priority $\wp(t+1)$ in next iteration, which is unknown at iteration $t$. To address this issue, this study estimates the upper bound of $d H(\widetilde{\wp}(t)) d^{T}$ so that we can find lower bound of $\gamma_{B}(t)$.

Since $q_{m}(\cdot)$ is a sigmoid function (after approximation), a maximum first-order derivative exists, 
denoted by $q_{\mathrm{m} \text {,max }}^{\prime}$. We further define

Also,

$$
\begin{gathered}
q_{\max }^{\prime} \triangleq \max _{m=1}^{M}\left\{q_{\text {m,max }}^{\prime}\right\} \\
n_{\max } \triangleq \max _{m=1}^{M}\left\{\bar{n}_{v}\right\} .
\end{gathered}
$$

$$
\begin{gathered}
\vartheta_{m}^{v}(t)=\min \left\{\beta_{q}^{v} p_{m}^{v}(t+1), \beta_{q}^{v} \wp_{m}^{v}(t)\right\}, \\
\vartheta_{\min }(t)=\min _{m=1}^{M}\left\{\min _{v=1}^{V}\left\{\vartheta_{m}^{v}(t)\right\}\right\} .
\end{gathered}
$$

Replacing $q_{m}^{\prime}, n$, and $\beta_{q}^{v} p_{M}^{v}$ by $q_{\max }^{\prime}, n_{\max }, \vartheta_{\min }(t)$ in Equation (40), we can see that the denominator of $\gamma_{B}(t)$ satisfy

$$
\frac{1}{2} d H(\widetilde{\wp}(t)) d^{T} \leq \frac{1}{2}\left[\frac{1}{\Delta \tau^{2}}\left(q_{\max }^{\prime}\right)^{2}\left(n_{\max }\right)^{2} V+\frac{1}{\vartheta_{\min }(t)}\right] \sum_{v=1}^{V} \sum_{m=1}^{M}\left(p_{m}^{v}(t+1)-\wp_{m}^{v}(t)\right)^{2} .
$$

Furthermore, by defining $\beta_{\max }=\max _{v=1}^{V}\left\{\beta_{q}^{v}\right\}$, it is clear that the nominator of $\gamma_{B}(t)$ satisfy

$$
\sum_{v=1}^{V} \frac{1}{2 \beta_{q}^{v}} \sum_{m=1}^{M}\left(p_{m}^{v}(t+1)-\wp_{m}^{v}(t)\right)^{2} \geq \frac{1}{2 \beta_{\max }} \sum_{v=1}^{V} \sum_{m=1}^{M}\left(p_{m}^{v}(t+1)-\wp_{m}^{v}(t)\right)^{2} .
$$

Plugging the boundaries given in Equations (50) and (51) into the last step of Equation (48), we know that the lower bound of $\gamma_{B}(t)$ is given below.

$$
\gamma_{B}(t) \geq \frac{1}{\beta_{\max }\left[\frac{1}{\Delta \tau^{2}}\left(q_{\max }^{\prime}\right)^{2}\left(n_{\max }\right)^{2} V+\frac{1}{\vartheta_{\min }(t)}\right]} .
$$

Thus, we can have lemma 2 below.

Lemma 2: Taking the step size

$$
\gamma(t)=\frac{0.99}{\beta_{\max }\left[\frac{1}{\Delta \tau^{2}}\left(q_{\max }^{\prime}\right)^{2}\left(n_{\max }\right)^{2} V+\frac{1}{\vartheta_{\min }(t)}\right]},
$$

then $Z(p)$ is a descent function for the SDA.

Furthermore, the map: $\wp(t) \rightarrow \wp(t+1)$ is defined as the composite map $A=B C$, where $B: \wp(t) \rightarrow$ $p(t+1)$ is defined by Equation (28), which is a continuous map; $C: p(t+1) \rightarrow \wp(t+1)$ defined by Equation (29), which is also continuous map. Moreover, notice from equation (52) that in the definition of $\gamma(t)$, the only term that depends on $\widetilde{\S}(t)$ is $\vartheta_{\min }(t)$. On the other hand, by the definition of $\vartheta_{\min }(t)$ in (49), we can see that $\vartheta_{\min }(t)$ is continuous in $\widetilde{\wp}(t)$, thanks to the continuity of the min operator. Therefore, according to Theorem 7.3.2 in Bazaraa et al. (2006) and its Corollary 2, we claim Lemma 3 below.

Lemma 3: The map $\wp(t) \rightarrow \wp(t+1)$ is a closed map.

Given Lemmas 1, 2 and 3, this study concludes the proof of the convergence of the SDA by Theorem 3.

Theorem 3: The SDA converges to the equilibrium of the MGS, given the step size $\gamma(t)$ is defined by Equation (52).

\section{Numerical Experiments}

Numerical experiments are conducted to validate the applicability and efficiency of the proposed parking mechanism. More exactly, the experiments have two objectives: (i) whether the proposed parking mechanism and algorithm are applicable in terms of computational performance; (ii) whether the proposal parking mechanism can improve the parking efficiency from the perspectives of both system and users. In this section, we first present our experiment setup and design, and then discuss the obtained insights.

6.1. Experiment setup and design

The road network from Guicheng Community, Nanhai District, Foshan, China is used as the testbed to setup our experiments (See Figure 1). The road network contains 113 nodes and 210 links. There are 25 parking lots in total in this research area. According to the transportation plan of Nanhai District, the road 
network of the test-bed is separated into 11 traffic analysis zones (TAZs). Accordingly, this study collects the necessary data for the road network, parking lots, and parking demands, respectively.

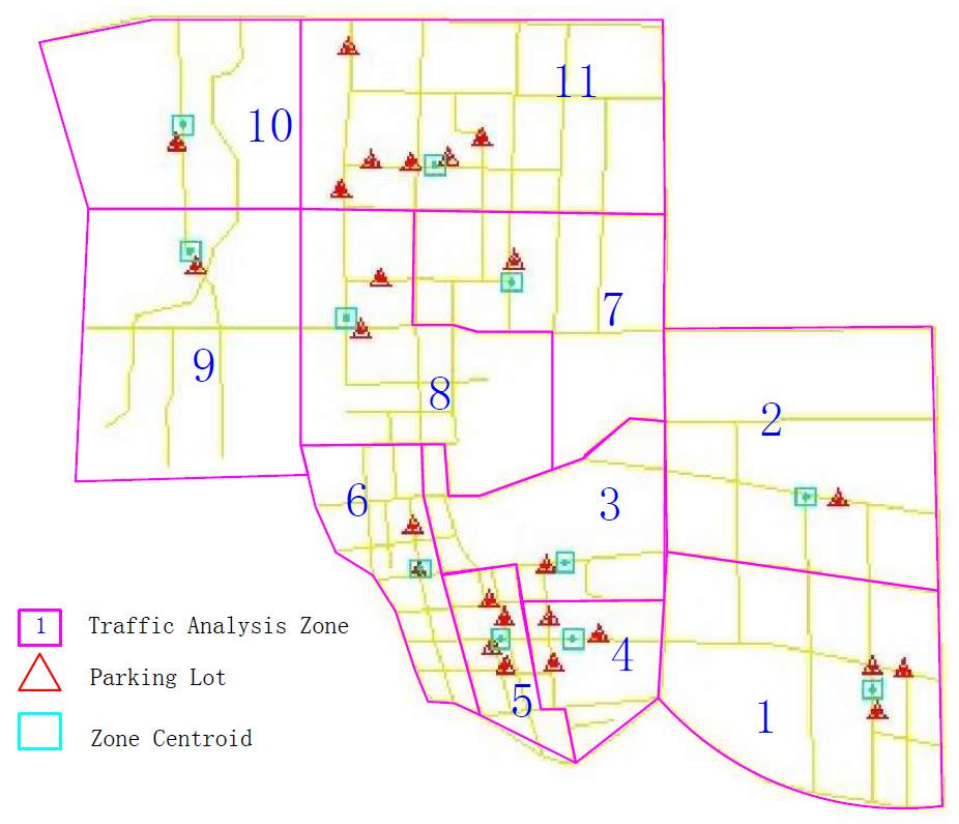

Figure 1. Road network, parking lots and TAZs

Below presents the detailed description for these data. (i) The road network data include the network topology data, the TAZs and the centroid location of each TAZ. (ii) The operational data for all parking lots were collected during 8 AM to7 PM on March 27th, 2012. For each parking lot, the collected operational data including parking lot capacity, average parking time, hourly arrival rate, hourly occupancy and parking fee. (iii) The parking demand data approximated by the parking demand in each TAZ. Specifically, due to the lack of detailed information about the origin and destination of individual travelers, this study sets the zone centroid of a TAZ as the common destination of all the travelers with their destinations in that zone. Accordingly, the parking demands over the testbed are grouped into different types of parking customers $\{v\}$ according to the TAZs. Namely, all travelers with their destinations in the same TAZ belong to one type of parking customers. The parking demand in each TAZ obeys Poisson distribution with the average hourly arrival rate equal to the aggregated rate across all the parking lots in this TAZ. Correspondingly, the shortest walking distance from a parking lot to the corresponding TAZ centroid represents the walking distance in a trip.

The setup of the experiment is specified as follows. (i) The experiment is run in 66 different scenarios, addressing the parking demands from $8 \mathrm{AM}$ to $7 \mathrm{PM}$ with the time interval $\Delta \tau=10 \mathrm{mins}$. It means that the parking strategy for each type of travelers is generated in every 10 minutes. (ii) During each $\Delta \tau$, the average number of the arriving travelers in each type, $\bar{n}_{v}$, equals to the time interval multiplying the hourly arrival rate given. Similarly, the departure rate $\mu_{m}$ is calculated by the reciprocal of the average parking time. (iv) The constant walk distance $s_{m}^{v}$ and parking $\operatorname{cost} C_{m}^{v}$ are given as constant values. (v) The weights of the three factors, $q_{m}^{v} s_{m}^{v}$, and $C_{m}^{v}$, in the utility function are randomly selected according to their magnitude so that none of them will dominate the decision. Even though various combinations of the weights are applied to test the performance of strategies, we obtain similar observations. As an example, blew presents the results with the weights: $\beta_{q}^{v}=3000, \beta_{s}^{v}=1$ and $\beta_{C}^{v}=200$. The SDA algorithm is implemented in MATLAB 7.1 and run on a computer with processor: Intel(R) Core(TM) i7-3770 CPU @ 3.40GHz and RAM: 8.0 GB. Note that a large value, such as 3000 and 200, are assigned to the weight of $q_{m}^{v}$ and $\beta_{C}^{v}$ so that we can avoid overweighting each individual factor by making the three values are in the same magnitude. More exactly, if walking distance or parking price dominates the solution, the DCPM will end 
with the same solution to the nearest first (or cheapest first) strategy since these two factors won't change with the parking decision. The effect of the DCPM is hard to see. On the other hand, if we weight $q_{m}$ too much in the utility function, then we will see the significant benefit of DCPM but the effect of walking distance and parking distance in parking decision is covered. To give a more comprehensive comparison, this study assigned the weights to the three factors in the utility function so that they are different values within the same magnitude.

\subsection{Examining the Computational Performance}

This study first exams the computational performance of the SDA in terms of the convergence and computation efficiency. Our experiment results (such as the examples presented in Figure 2) indicate that the descent function (i.e. the objective function of the model MP in Equation (9)) strictly decreases along with the algorithm iterations in all the experimental scenarios. Thus, we conclude that the SDA converges consistently in all scenarios. The experiment results are consistent to our theoretical proof. It is also observed that the SDA converges quickly. It takes 2-5 seconds to reach the coverage solution in each scenario, with the mean equal to $2.8106 \mathrm{~s}$ and the variance equal to 0.2529 over all scenarios. Overall, we conclude that the computational performance of the SDA satisfies the online application.

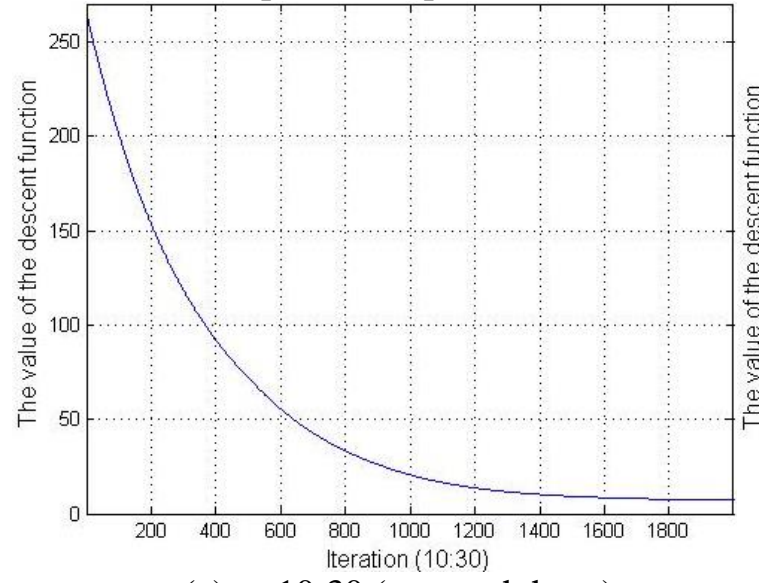

(a) $\mathrm{t}=10: 30$ (non-rush hour)

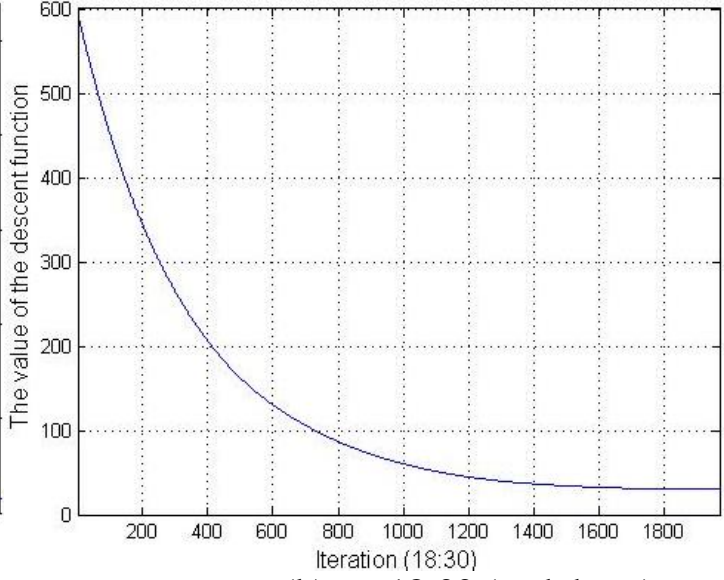

(b) $\mathrm{t}=18: 00$ (rush hour)

Figure 2. Illustration of the convergence of the SDA algorithm

\subsection{Validating the Efficiency of the DCPM}

This study thus validates the efficiency of the DCPM through comparing the performance of the proposed DCPM to three greedy strategies. (i) $G S_{w}$, the nearest first strategy, by which a traveler detects the availability of the parking facility in an ascending order according to the walking distance. (ii) $G S_{c w}$, the cheapest first strategy, by which a traveler first detects the availability of the parking facilities in an descending order according to the parking price, given the parking facilities within $1 \mathrm{~km}$ walking distance. If fails, the traveler follows the nearest first strategy to detect the availability of the parking facilities outside $1 \mathrm{~km}$ walking distance. (iii) $G S_{o w}$, the least cruise first strategy, by which a traveler detects the availability of the parking facilities within $1 \mathrm{~km}$ in a descending order of the number of real-time open spots. If fails, the traveler detects the availability of parking facilities with walking distance greater than $1 \mathrm{~km}$ still following the least cruise policy. (iv) The DCPM, by which a parking customer will choose the parking lot according to the priority obtained in the equilibrium parking decision. Note that $G S_{o w}$ and the DPM assume that vehicles have the access to real-time information about the open spots at parking facilities. Vehicles following the DCPM or $G S_{\text {ow }}$ respond to the real-time information independently with or without coordination. Vehicles following $G S_{w}$ or $G S_{c w}$ do not receive real-time information. The performances of the parking strategies are compared from four perspectives. (i) $N_{c}$, the number of travelers need to cruise (i.e., the number of travelers fails to find the parking spot in the first attempt). (ii) $L_{c}$, the average cruise distance per traveler to find an available parking lot. (iii) $L_{w}$, the average walking distance per traveler. (iv) 
$F_{p}$, the average parking fee per traveler. The experiment results are given in Figure 3 - Figure 6. Comprehensive discussions are given below.

Figure 3 presents the results about $N_{c}$. It indicates that almost in all scenarios, $N_{c}$ under the strategy of the DCPM is much smaller than the corresponding values under the other three greedy strategies. Especially, in the peak hour 18:00, the value of $N_{c}$ under the DCPM is significantly reduced ( $N_{c}=13$ under the DCPM, $N_{c}=62$ under the $G S_{w}, N_{c}=64$ under the $G S_{c w}$, and $N_{c}=55$ under $G S_{o w}$ in Figure 3) Similar observations can be obtained as we exam the value of $L_{c}$ shown in Figure 4.

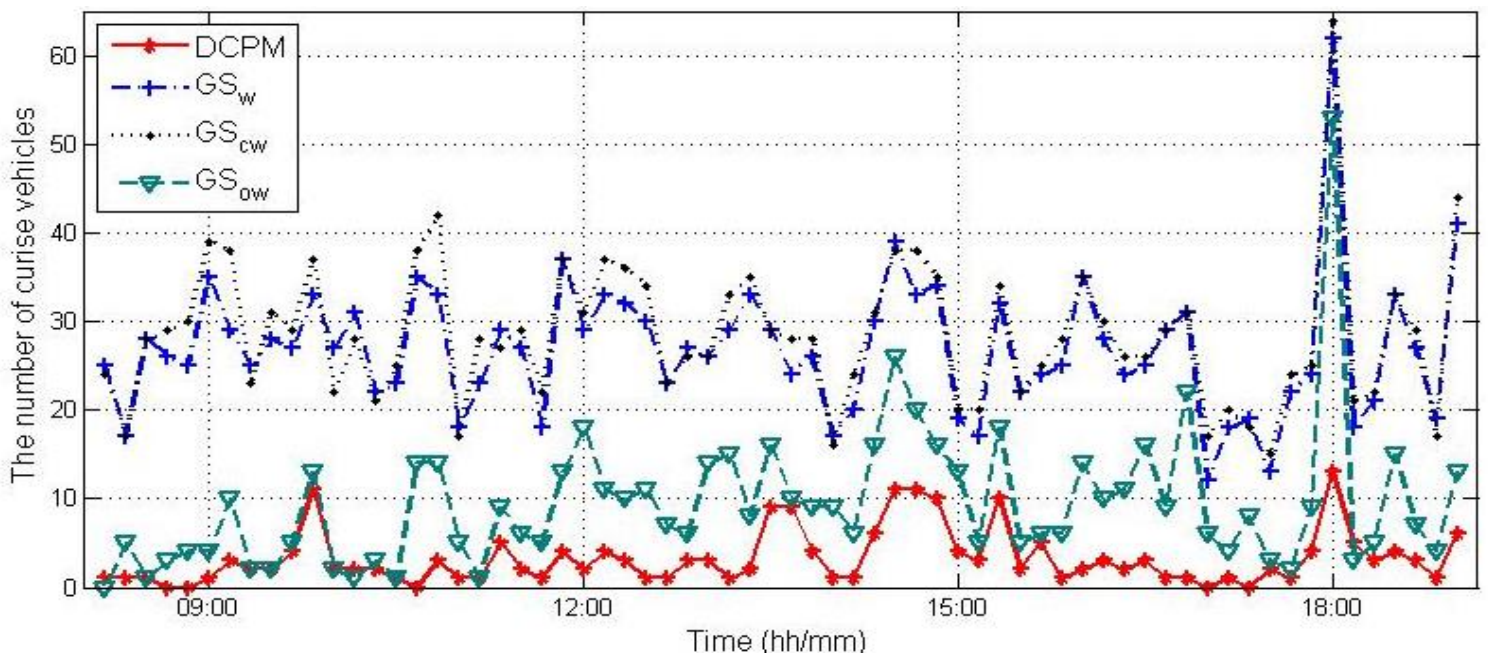

Figure 3. The number of cruise travelers under different parking guiding strategies

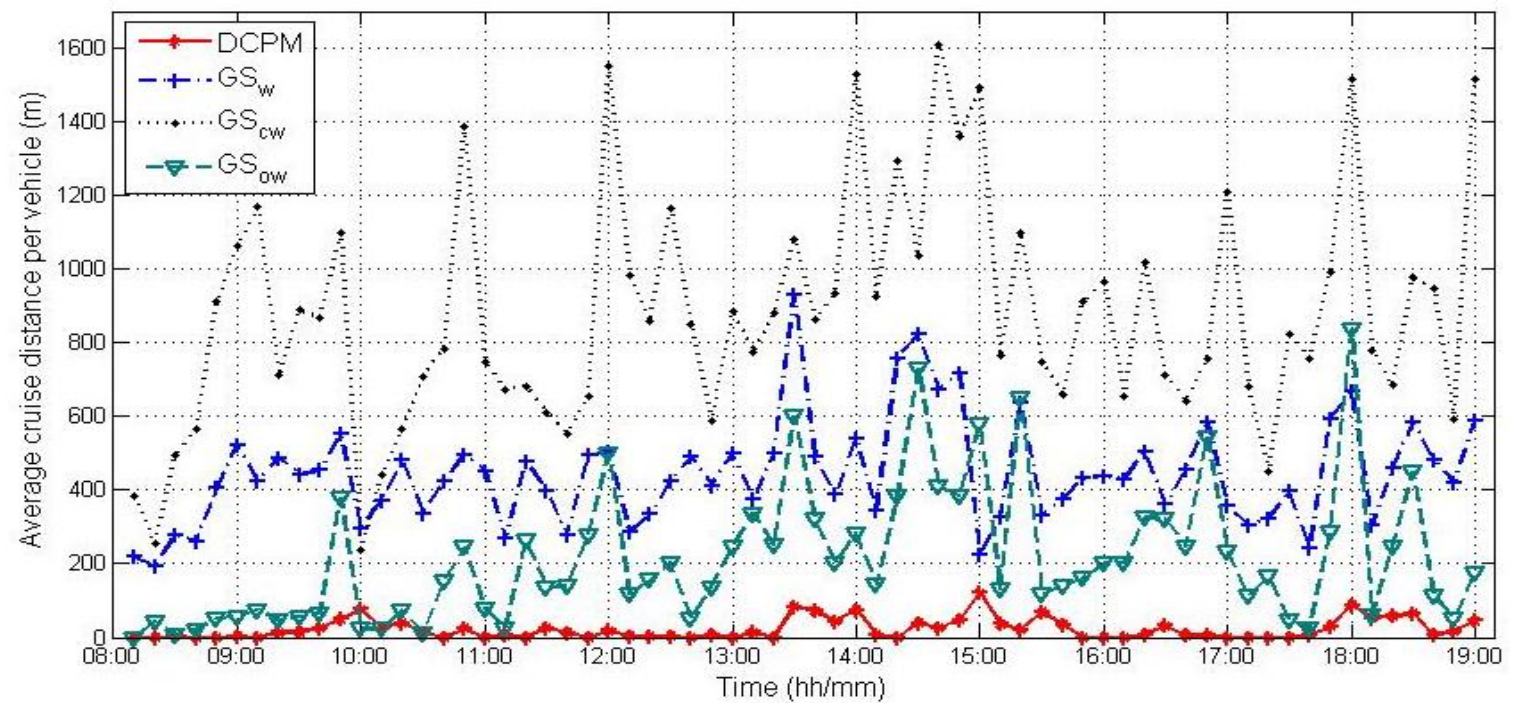

Figure 4 . The average cruise distance per traveler under different parking guiding strategies

Table 1 presents the average performance of different strategies in terms of $N_{c}$ and $L_{c}$ across all scenarios $(8 \mathrm{am}-7 \mathrm{pm})$ in our experiments. It indicates that the DCPM can reduce the average number of cruise travelers by $88.06 \%, 88.75 \%$ and $65.70 \%$ respectively as compared to the strategies of $G S_{w}, G S_{c w}$, and $G S_{o w}$. Correspondingly, the average cruise distance will decrease $95.00 \%, 95.47 \%$ and $89.63 \%$ respectively by applying the DCPM instead of the other three strategies. In addition, it is observed that $G S_{o w}$ performs worse than the DCPM, but better than the $G S_{w}$ and $G S_{c w}$ strategies in terms of $N_{c}$ and $L_{c}$. Thus, we claim that the real-time information about the number of the open spots will help in reducing $N_{c}$ and $L_{c}$ comparing to the strategies without real-time information. The DCPM will improve the benefit of the real-time information further. The experiment results in Figure 3, Figure 4 and Table 1 demonstrate that the DCPM will significantly reduce parking congestion at the parking facilities. 
Table 1. Performance of $N_{c}$ and $L_{c}$

\begin{tabular}{|c|c|c|c|c|}
\hline \multirow{2}{*}{ Parking Strategy } & \multicolumn{2}{|c|}{ Number of cruise vehicles } & \multicolumn{2}{c|}{ Average cruise distance (m) } \\
\cline { 2 - 5 } & $N_{c}$ & Relative difference & $L_{c}$ & $\begin{array}{c}\text { Relative } \\
\text { difference }\end{array}$ \\
\hline DCPM & 3.2121212 & -- & 22.2002789 & -- \\
\hline$G S_{w}$ & 26.893939 & $-88.06 \%$ & 444.429013 & $-95.00 \%$ \\
\hline$G S_{c w}$ & 28.545454 & $-88.75 \%$ & 878.355461 & $-97.47 \%$ \\
\hline$G S_{\text {ow }}$ & 9.3636363 & $-65.70 \%$ & 214.123135 & $-89.63 \%$ \\
\hline
\end{tabular}

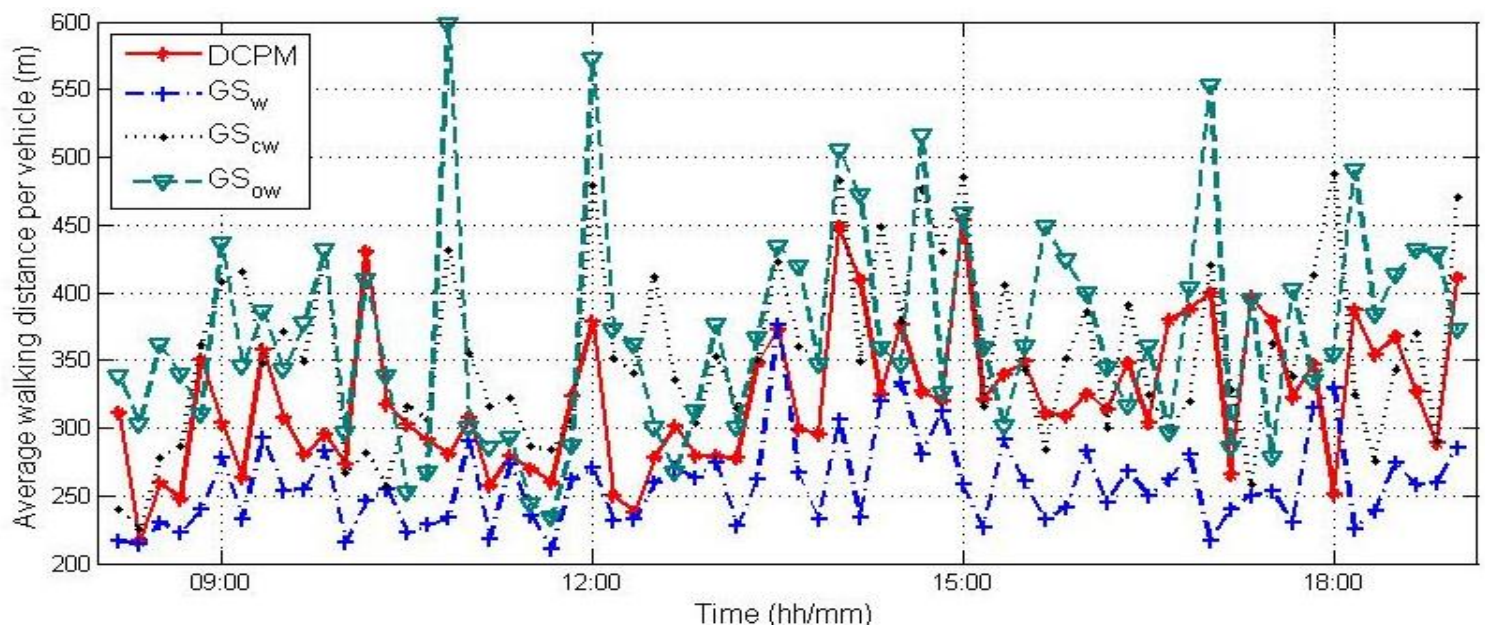

Figure 5. The average walking distance per vehicle under different parking guiding strategies

Figure 5 presents the experiment results about the average walking distance per traveler, $L_{w}$ under each scenario. It shows that the values of $L_{w}$ under the DCPM are greater than the nearest first strategy $G S_{w}$ focusing on minimizing walking distance, but are smaller than the cheapest first strategy $G S_{c w}$ focusing on minimizing parking cost and also considering the walking distance as a side factor. On the other hand, we find that $G S_{\text {ow }}$ performs worse than all other strategies. Thus, we conclude that each vehicle independently response to real time information about open spots at parking facilities may lead to higher walking distance. The DCPM will improve the benefit of the real-time information.

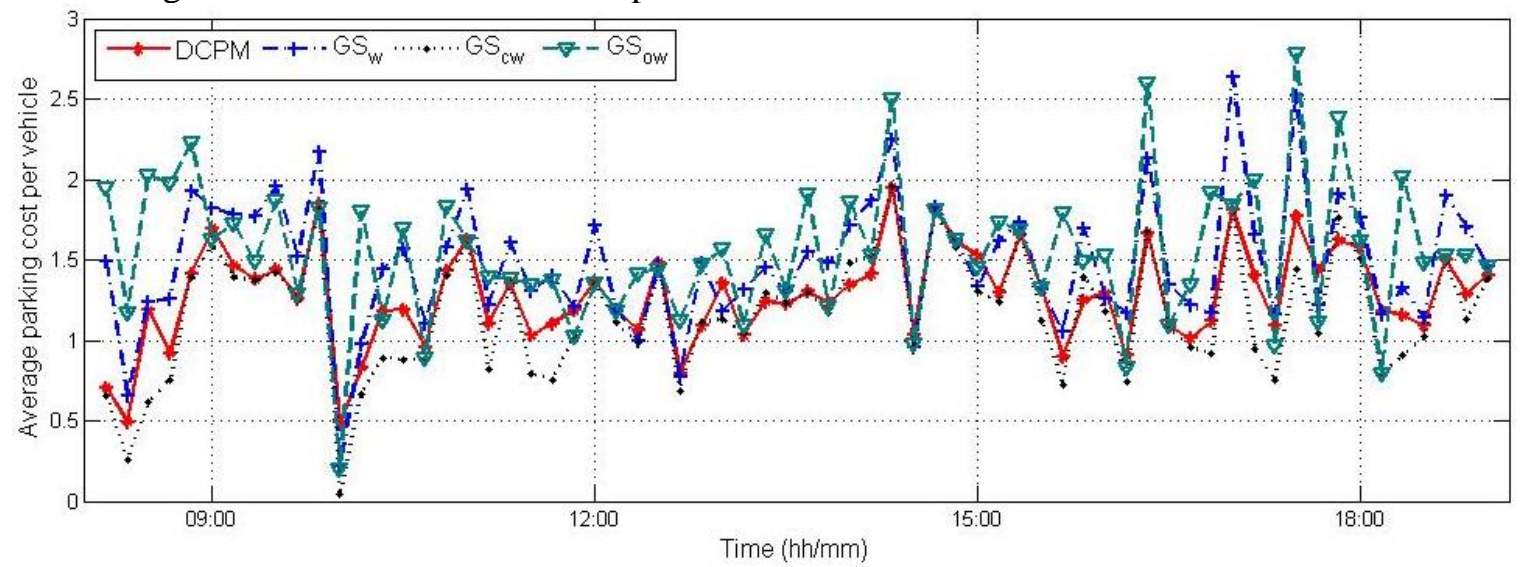

Figure 6 . The average parking cost per vehicle under different parking guidance

Figure 6 compares the average parking fee $F_{p}$ under different parking guidance over all the scenarios 
in the experiments. It is demonstrated that the values of $F_{p}$ under the DCPM are a little bit larger than the values under the $G S_{c w}$, but are smaller than the greedy strategies of $G S_{w}$ and $G S_{o w}$.

Table 2. The average walking distance and average parking fee

\begin{tabular}{|c|c|c|c|c|}
\hline \multirow{2}{*}{ Strategy } & \multicolumn{2}{|c|}{ Average walking distance } & \multicolumn{2}{c|}{ Average parking cost } \\
\cline { 2 - 5 } & $L_{w}$ & Relative difference & $F_{p}$ & Relative difference \\
\hline DCPM & 321.4104 & -- & 1.274916 & -- \\
\hline$G S_{w}$ & 258.1392 & $24.51 \%$ & 1.482975 & $-14.03 \%$ \\
\hline$G S_{c w}$ & 352.5098 & $-8.82 \%$ & 1.161707 & $9.75 \%$ \\
\hline$G S_{\text {ow }}$ & 368.3646 & $-12.75 \%$ & 1.547670 & $-17.62 \%$ \\
\hline
\end{tabular}

Last, Table 2 provides the summary of the insights obtained from the experiments regarding to the walking distance $L_{w}$ and parking cost $F_{p}$. More exactly, the DCPM will increase $24.51 \%$ of the average waking distance but reduce $14.03 \%$ of the average parking cost as compared to the $G S_{w}$ strategy. On the other hand, the DCPM will increase $9.75 \%$ of average parking cost but reduce $8.82 \%$ of the average walking distance from $G S_{c w}$ strategy. When compared to the strategy of $G S_{\text {ow }}$, the DCPM will reduce both $17.62 \%$ of average parking cost and $12.75 \%$ of the average walking distance. Hence, we conclude that the DCPM provides a trade-off solution to the greedy strategies of $G S_{w}$ and $G S_{c w}$ giving priority to $L_{w}$ and $\mathrm{F}_{\mathrm{p}}$ respectively, but performs better than $\mathrm{GS}_{\mathrm{ow}}$ in both $\mathrm{L}_{\mathrm{w}}$ and $\mathrm{F}_{\mathrm{p}}$ perspectives. It is also noticed that the cruise least strategy performs worst among all the strategies in terms of $L_{w}$ and $F_{p}$. It again confirms that letting individual vehicle independently respond real-time information is not an efficient strategy to address parking congestion.

Overall, the numerical experimental results confirm that the proposed DCPM can efficiently provide parking guidance to a group of travelers under a distributed computation framework, sustaining both system performance and individual travelers' parking preference.

\section{Conclusion}

This research proposes a decentralized and coordinated online parking mechanism. It seeks to reduce parking congestion at multiple parking facilities in a CBD through mitigating the competition among vehicles. To establish this DCPM, this study considers the vehicles searching parking spaces in a CBD during a time interval as one parking coordination group, and formulates the competitions among parking vehicles en route by stochastic Poisson game defined by a three-tuples: type of players; actions, and expected utility function. Specifically, this study considers travelers with same or close destinations share the same parking priority, thus treats them as one type of travelers in the game. A deterministic number of types are considered in the game model, but the number of travelers in each type follows a Poisson distribution. The action set of travelers in the game includes all the parking facilities in consideration. The expected utility function mainly factors the probability that an arriving traveler is forced to cruise. It is formulated by using $\mathrm{M} / \mathrm{M} / \mathrm{c}$ queue theory given the parking time of each vehicle obeys exponential distribution and the number of travelers arriving to a parking facility follows Poisson distribution. Given travelers' parking choice behavior is modeled by multinomial logit discrete choice model, this study proposes the equilibrium condition for the proposed stochastic Poisson game, and also proves that the stochastic Poisson game is a potential game and has an unique equilibrium. Furthermore, a simultaneously updating distributed algorithm is developed to search the equilibrium solution of the DCPM. Its convergence is proved by both mathematical analysis and numerical experiments.

The road network covering Guicheng Community, Nanhai District, Foshan, China is used as the testbed to conduct numerical experiments. Field parking operational data collected from $7 \mathrm{am}-8 \mathrm{pm}$ on March $27^{\text {th }}, 2012$ were collected and used to perform the validation experiments. Specifically, the proposed DCPM performs parking guidance for the arriving travelers to the CBD every 10 minutes, thus the experiments 
include 66 scenarios in total. Under each scenario, the performance of the proposed the DCPM is compared to three greedy strategies following the nearest first, cheapest first, and least cruise first polices. Our experiment results indicate that the DCPM significantly reduces the number of cruise vehicles $\left(N_{c}\right)$ as well as the average cruise distance $\left(L_{c}\right)$ as it is compared to the three greedy strategies. This advantage is more significant during rush hours. In addition, it is observed that the least cruise strategy using real-time information performs worse than the DCPM, but better than the nearest and cheapest first strategies in perspectives of $N_{c}$ and $L_{c}$. Thus, we claim that the real-time information about the number of the open spots helps in reducing $N_{c}$ and $L_{c}$ compared to the strategies without real-time information. The DCPM further improves the benefit of the real-time information. Last, the DCPM provides a trade-off solution to the nearest first and cheapest first strategies giving priority to walking distance $\left(L_{w}\right)$ and parking cost $\left(F_{p}\right)$ respectively, but performs better than the cruise least strategy in both $L_{w}$ and $F_{p}$. The cruise least strategy performs worst among all the strategies in terms of $L_{w}$ and $F_{p}$. It again confirms that letting individual vehicles independently respond to real-time information is not an efficient strategy to address parking congestion. Overall, the proposed DCPM can reduce the competition among vehicles on parking resources and efficiently guidance parking decisions.

The immediate extension of the proposed research is to involve the network travel time variation into the utilities so that the parking decisions and coordination among vehicles can be made further away from their destinations. This new idea will involve the competition of vehicles in both road resources and parking resources and make the game model reflect reality better, but will also initiate the challenges for modeling process, such as the formation of the coordination group, the formulation of the expected utility function, etc. We will explore these challenges in our future research.

Acknowledgement: The research presented in this paper is partially supported by NSF award CMMI 1436786. The authors are very thankful to Dr. Peng Xu in College of Civil and Transportation Engineering at Hohai Univsity for providing the field data used in the numerical experiments. The authors are also very grateful to the two anonymous reviewers, whose comments have improved the presentation of the paper greatly.

\section{Reference}

Asakura, Y., Kashiwadani, M., 1994. Effects of parking availability information on system performance: a simulation model approach. In Vehicle Navigation and Information Systems Conference, 1994, pp. 251254. IEEE.

Ayala, D., Wolfson, O., Xu, B., Dasgupta, B., Lin, J., 2011. Parking slot assignment games. In Proceedings of the 19th ACM SIGSPATIAL International Conference on Advances in Geographic Information System, pp. 299-308. ACM.

Ayala, D., Wolfson, O., Xu, B., DasGupta, B., Lin, J., 2012a. Parking in competitive settings: A gravitational approach. In Mobile Data Management (MDM), 2012 IEEE 13th International Conference on, pp. 27-32. IEEE.

Ayala, D., Wolfson, O., Xu, B., DasGupta, B., Lin, J., 2012b. Pricing of parking for congestion reduction. In Proceedings of the 20th International Conference on Advances in Geographic Information Systems, pp. 43-51. ACM.

Bazaraa, M.S., Sherali, H.D., Shetty, C.M., 2006. Nonlinear Programming: Theory and Algorithms. John Wiley \& Son, Inc.

Borwein, J. M.,. Lewis, A. S., 2006. Convex analysis and nonlinear optimization: Theory and examples. New York: Springer.

Caliskan, M., Graupner, D., Mauve, M., 2006, September. Decentralized discovery of free parking places. In Proceedings of the 3rd international workshop on Vehicular ad hoc networks, pp. 30-39. ACM.

Chen, Z., Yin, Y., He, F. and Lin, J.L., 2015. Parking reservation for managing downtown curbside parking. Transportation Research Record. Vol. 2498, 12-18 
Delot, T., Cenerario, N., Ilarri, S., Lecomte, S., 2009, September. A cooperative reservation protocol for parking spaces in vehicular ad hoc networks. In Proceedings of the 6th International Conference on Mobile Technology, Application \& Systems, p. 30. ACM.

Delot, T., Ilarri, S., Lecomte, S., Cenerario, N., 2013. Sharing with caution: Managing parking spaces in vehicular networks. Mobile Information Systems, 9(1), 69-98.

Du, L., L.S. Han, and X.Y. Li. (2014). Distributed coordinated in-vehicle online routing using mixedstrategy congestion game. Transportation Research Part B: Methodological 67, 1-17, 2014.

Ellis, R. H., Rassam, P. R., Bennett, J. C., 1972. Development and implementation of a parking allocation model. Highway Research Record, (395).

Geng, Y., Cassandras, C. G., 2013. New "Smart Parking” System Based on Resource Allocation and Reservations. Intelligent Transportation Systems, IEEE Transactions on, 14(3), 1129-1139.

Gross, D., Shortle, J.F., Thompson, J.M., and Harris, C.M., 2008. Fundamentals of queueing theory. $4^{\text {th }}$ Edition, John Wiley \& Sons.

Guo, L., Huang, S., Zhuang, J., Sadek, A., 2013. Modeling parking behavior under uncertainty: a static game theoretic versus a sequential neo-additive capacity modeling approach. Networks and Spatial Economics 13, 327-350

He, F., Yin, Y., Chen, Z., Zhou, J., 2015. Pricing of parking games with atomic players. Transportation Research Part B: Methodological, 73, 1-12.

Idris, M. Y. I., Leng, Y. Y., Tamil, E. M., Noor, N. M., Razak, Z., 2009. Car park system: a review of smart parking system and its technology. Information Technology Journal, 8(2), 101-113.

Jung, T., Mao, X., Li, X.-Y., Tang, S., Gong, W., Zhang, L., 2013. Privacy-preserving data aggregation without secure channel: Multivariate polynomial evaluation. In: INFOCOM, IEEE, pp. 2634-2642.

Kokolaki, E., Karaliopoulos, M., Stavrakakis, I., 2014. On the efficiency of information-assisted search for parking space: A game-theoretic approach. In Self-Organizing Systems, pp. 54-65. Springer Berlin Heidelberg.

Lam, W.H., Li, Z.C., Huang, H.J., Wong, S.C., 2006. Modeling time-dependent travel choice problems in road networks with multiple user classes and multiple parking facilities. Transportation Research Part B: Methodological, 40(5), 368-395.

Lambe, T. A., 1996. Driver choice of parking in the city. Socio-Economic Planning Sciences, 30(3), 207219.

Li, Z. C., Huang, H. J., Lam, W. H., Wong, S. C., 2007. A model for evaluation of transport policies in multimodal networks with road and parking capacity constraints. Journal of Mathematical Modelling and Algorithms, 6(2), 239-257.

Liu, J., Chen, R., Chen, Y., Pei, L., Chen, L., 2012. iParking: An intelligent indoor location-based smartphone parking service. Sensors, 12(11), 14612-14629.

Mackowski, D., Bai, Y., Ouyang, Y., 2015. Parking Space Management via Dynamic Performance-Based Pricing. arXiv preprint arXiv:1501.00638.

Mejri, N., Ayari, M., Langar, R., Kamoun, F., Pujolle, G., Saidane, L., 2014. Cooperation versus competition towards an efficient parking assignment solution. In Communications (ICC), 2014 IEEE International Conference on, pp. 2915-2920. IEEE.

Myerson, R.B., 1998. Population uncertainty and Poisson games. International Journal of Game Theory 27(3): 375-392.

Monderer, D. and Shapley, L., 1996. Potential Games. Games and Economic Behavior 14: 124-143.

Nelson, B. L. 1995. Stochastic Modeling: Analysis and Simulation. New York: McGraw-Hill.

Schlote, A., King, C., Crisostomi, E. and Shorten, R., 2014. "Delay-tolerant stochastic algorithms for parking space assignment." Intelligent Transportation Systems, IEEE Transactions on 15(5): 1922-1935. 
Sheffi, Y., 1985. Urban transportation networks: equilibrium analysis with mathematical programming methods, Prentice Hall.

Shoup, D. C., 2006. Cruising for parking. Transport Policy 13(6): 479-486.

Teodorović, D., Lučić, P., 2006. Intelligent parking systems. European Journal of Operational Research, 175(3), 1666-1681.

Thompson, R. G., Richardson, A. J., 1998. A parking search model. Transportation Research Part A: Policy and Practice, 32(3), 159-170.

Yan, G., Yang, W., Rawat, D. B., Olariu, S., 2011. SmartParking: A secure and intelligent parking system. Intelligent Transportation Systems Magazine, IEEE, 3(1), 18-30.

Zou, B. , Kafle, N., Wolfson, O., Lin, J., 2015. A Mechanism design based approach to solving parking slot assignment in the information era. Transportation Research Part B: Methodological, forthcoming.

\section{Appendix A: Sample-Regression for the Approximation function}

This study develops the sigmoid function in Equation (39) through a sample-regression method presented below. More specifically, this research first samples $q(\lambda)$ at a given number of points within certain range of interests. Then a regression is ran to determine the values of $b$ and $a$ to minimize the error on the sample. In this particular research, the range of interests is $[0, \mu)$, where $\delta \geq 1$ is a constant value. Suppose we evenly sample $K$ points within $[0, \mu]$, we obtain a sample of size $K$, denoted by $\left(x_{k}, y_{k}\right)_{k=1, \cdots, K}$. That is to say:

$$
\begin{gathered}
w_{k}=(k-1) \frac{\delta \mu}{K}, \quad k=1, \cdots, K, \\
y_{k}=q\left(x_{k}\right), \quad k=1, \cdots, K .
\end{gathered}
$$

With the sample, the next step is to determine the values of $b$ and $a$. Note that $\hat{q}(\lambda)$ is a nonlinear function of $b$ and $a$, in principle, a nonlinear regression is needed. However, nonlinear regressions are normally challenging. Therefore this research propose to perform transformations so that the values of $b$ and $a$ can be determined by a linear regression. In particular, it is noticed that

$$
\hat{q}(\lambda)=\frac{1}{1+b e^{a \lambda}} \Leftrightarrow \frac{1}{\hat{q}(\lambda)}-1=b e^{a \lambda} \Leftrightarrow \ln \left(\frac{1}{\hat{q}(\lambda)}-1\right)=\ln b+a \lambda .
$$

Hence, we first apply transformations on the samples as follows:

$$
\begin{gathered}
\widehat{w}_{k}=w_{k}, \quad k=1, \cdots, K, \\
\hat{y}_{k}=\ln \left(\frac{1}{y_{k}}-1\right), \quad k=1, \cdots, K .
\end{gathered}
$$

After the transformation, a transformed sample set $\left(\widehat{w}_{k}, \widehat{y}_{k}\right)_{k=1, \cdots, K}$ is obtained. By running a linear regression with an intercept on the transformed sample set, we obtain an estimated intercept $\hat{b}$ and and a coefficient $\hat{a}$. In fact, closed form formula for $\hat{b}$ and $\hat{a}$ are given by:

$$
\begin{gathered}
\hat{a}=\frac{1}{C}\left[-\left(\sum_{k=1}^{K} \widehat{w}_{k}\right)\left(\sum_{k=1}^{K} \hat{y}_{k}\right)+K \sum_{k=1}^{K} \widehat{w}_{k} \hat{y}_{k}\right] \\
\hat{b}=\frac{1}{C}\left[\left(\sum_{k=1}^{K} \widehat{w}_{k}^{2}\right)\left(\sum_{k=1}^{K} \hat{y}_{k}\right)-\left(\sum_{k=1}^{K} \widehat{w}_{k}\right)\left(\sum_{k=1}^{K} \widehat{w}_{k} \hat{y}_{k}\right)\right] .
\end{gathered}
$$

Notice that $w_{1}<w_{2}<\cdots<w_{k}$, hence $y_{1}<y_{2}<\cdots<y_{k}$, which in turn implies that $\hat{y}_{1}>\hat{y}_{2}>\cdots>\hat{y}_{k}$, by Chebyshev's sum inequality we derive that

$$
\frac{1}{K} \sum_{k=1}^{K} \widehat{w}_{k} y_{k}<\left(\frac{1}{K} \sum_{k=1}^{K} \widehat{w}_{k}\right)\left(\frac{1}{K} \sum_{k=1}^{K} \hat{y}_{k}\right) \Rightarrow K \sum_{k=1}^{K} \widehat{w}_{k} \hat{y}_{k}<\left(\sum_{k=1}^{K} \widehat{w}_{k}\right)\left(\sum_{k=1}^{K} \hat{y}_{k}\right) \Rightarrow \hat{a}<0
$$

Equation (53) ensures that the regression will provide us a negative value of $\hat{a}$. Let $a=\hat{a}, b=e^{\hat{b}}$, we obtain the values of $b$ and $a$. Note that we must have $a<0, b>0$. 
This study presents an example to demonstrate this approximation, using the data in our numerical analyses. Specifically, we evenly sample $K=1500$ points within $\left[0,1.5 \mu_{m}\right]$, c is set as a parameter changing from 1 to 30 . Note that $y_{k}=0.99999$ as the sample falls in the range of $\left[\mu_{m}, 1.5 \mu_{m}\right]$ since $\hat{y}_{k}=$

$\ln \left(\frac{1}{y_{k}}-1\right)$ is negative infinity for $y_{k}=1$. Based on the sample data, we conduct the regressions under different input values of $c$. Our regression results show that the coefficient of determination $R^{2}$ equals to 0.8118 on the average over all the experiments with the variance 0.00277 . In addition, the minimum and maximum values of $R^{2}$ equal to 0.8798 as $\mathrm{c}=7$ and 0.721 as $\mathrm{c}=30$, respectively. Thus, the approximation is reasonably good.

\section{Appendix B: Equation (28) in (Du et al, 2014)}

Given $p_{t+1}^{i}=\frac{e^{\beta C_{t+1}^{i}}}{\sum_{i=1}^{K} e^{\beta c_{t+1}^{i}}}, i=1, \ldots, K,\left\{p_{t+1}^{i}\right\}_{i=1}^{K}$ is the optimal solution of the mathematical model with decision variables $\left\{q_{t+1}^{i}\right\}_{i=1}^{K}$.

$$
\begin{aligned}
& \min \sum_{i=1}^{K} C_{t}^{i} q_{t+1}^{i}+\frac{1}{\beta_{v}} q_{t+1}^{i} \ln q_{t+1}^{i} \\
& \text { s.t. } \sum_{i=1}^{K} q_{t+1}^{i}=1 \\
& \qquad q_{t+1}^{i} \geq 0, \forall i=1, \ldots, K
\end{aligned}
$$

\section{Appendix C: Theorem 11 given by Borwein and Lewis (2006)}

Theorem 11. If the vector $q, p>0$ satisfies $\sum_{1}^{n} p_{i}=1$ and $\sum_{1}^{n} q_{i}=1$, then it holds that

$$
\sum_{1}^{n} p_{i} \log \frac{p_{i}}{q_{i}} \geq \frac{1}{2}\left(\sum_{1}^{n}\left|p_{i}-q_{i}\right|\right)^{2}
$$

Appendix D: Decomposition and Superposition of Poisson distribution (Myerson 1998, Nelson, 1995)

Superposition of a Poisson distribution

Let $\mathrm{X} \sim$ Poisson $\left(\eta_{1}\right)$ and $\mathrm{Y} \sim$ Poisson $\left(\eta_{2}\right)$. Suppose that $\mathrm{X}$ and $\mathrm{Y}$ are independent. Then, $\mathrm{X}+\mathrm{Y} \sim$ Poisson $\left(\eta_{1}+\eta_{2}\right)$.

Decomposition of a Poisson Process (distribution)

Consider a Poisson process $\{N(t): t \geq 0\}$ with rate $\eta$. Suppose that each time an event occurs it is classified as either a type I or a type II event. Suppose further that each event is classified as type I event with probability $\xi$ and as type II event with probability $1-\xi$. Let $N_{1}(t)$ denote respectively the number of type I events occurring in [0,t]. Let $N_{2}(t)$ denote respectively the number of type II events occurring in $[0, \mathrm{t}]$. Note that $N(t)=N_{1}(t)+N_{2}(t)$. Then, $\left\{N_{1}(t): t \geq 0\right\}$ and $\left\{N_{2}(t): t \geq 0\right\}$ are independent Poisson processes with respective rates $\eta_{1}=\xi p$ and $\eta_{2}=\eta(1-\xi)$. 